\title{
Comparison of complex networks and tree-based methods of phylogenetic analysis and proposal of a bootstrap method
}

Aristóteles Góes-Neto ${ }^{\text {Corresp.. }}{ }^{1}$ ， Marcelo V. C. Diniz ${ }^{2}$, Daniel S. Carvalho ${ }^{3}$, Gilberto C. Bomfim ${ }^{3}$, Ângelo A. Duarte $^{4}$, Jerzy A. Brzozowski ${ }^{5}$, Thierry C. Petit Lobao ${ }^{6}$, Suani T. R. Pinho ${ }^{7}$, Charbel N. El-Hani ${ }^{3}$, Roberto F. S. Andrade ${ }^{7}$

${ }^{1}$ Department of Microbiology, Universidade Federal de Minas Gerais, Brazil

2 Department of Microbiology, Universidade Federal de Minas Gerais, Belo Horizonte, Minas Gerais, Brazil

3 Institute of Biology, Universidade Federal da Bahia, Salvador, Bahia, Brazil

4 Department of Technology, Universidade Estadual de Feira de Santana, Feira de Santana, Bahia, Brazil

5 Interdisciplinary Graduate Program in Human Sciences, Federal University of Fronteira Sul, Erechim, Rio Grande do Sul, Brazil

6 Institute of Mathematics, Universidade Federal da Bahia, Salvador, Bahia, Brazil

${ }^{7}$ Institute of Physics, Universidade Federal da Bahia, Salvador, Bahia, Brazil

Corresponding Author: Aristóteles Góes-Neto

Email address: arigoesneto@icb.ufmg.br

Complex networks have been successfully applied to the characterization and modeling of complex systems in several distinct areas of Biological Sciences. Nevertheless, their utilization in phylogenetic analysis still needs to be widely tested, using different molecular data sets and taxonomic groups, and, also, by comparing complex networks approach to current methods in phylogenetic analysis. In this work, we compare all the four main methods of phylogenetic analysis (distance, maximum parsimony, maximum likelihood, and Bayesian) with a complex networks method that has been used to provide a phylogenetic classification based on a large number of protein sequences as those related to the chitin metabolic pathway and ATP-synthase subunits. In order to perform a close comparison to these methods, we selected Basidiomycota fungi as the taxonomic group and used a high-quality, manually curated and characterized database of chitin synthase sequences. This enzymatic protein plays a key role in the synthesis of one of the exclusive features of the fungal cell wall: the presence of chitin. The communities (modules) detected by the complex network method corresponded exactly to the groups retrieved by the phylogenetic inference methods. Additionally, we propose a bootstrap method for the complex network approach. The statistical results we have obtained with this method were also close to those obtained using traditional bootstrap methods. 
1 Comparison of complex networks and tree-based methods of phylogenetic analysis

4 Aristóteles Góes-Neto ${ }^{1 *}$, Marcelo V. C. Diniz ${ }^{1}$, Daniel S. Carvalho², Gilberto C. Bomfim², Ângelo A.

5 Duarte $^{3}$, Jerzy A. Brzozowski ${ }^{4}$, Thierry C. Petit Lobao ${ }^{5}$, Suani T. R. Pinho ${ }^{6}$, Charbel N. El-Hani², Roberto

$6 \quad$ F. S. Andrade 6.

$8{ }^{1}$ Universidade Federal de Minas Gerais, Institute of Biological Sciences, Belo Horizonte, MG, Brazil.

$9 \quad{ }^{2}$ Universidade Federal da Bahia, Institute of Biology, Salvador, BA, Brazil.

$10{ }^{3}$ Universidade Estadual de Feira de Santana, Department of Technology, Feira de Santana, BA, Brazil.

$11{ }^{4}$ Federal University of Fronteira Sul, Interdisciplinary Graduate Program in Human Sciences, Erechim,

12 RS, Brazil.

13 Universidade Federal da Bahia, Institute of Mathematics, Salvador, BA, Brazil.

$14{ }^{6}$ Universidade Federal da Bahia, Institute of Physics, Salvador, BA, Brazil.

$17{ }^{*}$ Corresponding author. Aristóteles Góes-Neto. Universidade Federal de Minas Gerais, Instituto de

18 Ciências Biológicas, Departamento de Microbiologia. Av. Antônio Carlos, 6627, Pampulha, Belo

19 Horizonte, MG, Brazil, CEP 31270-901. E-mail: arigoesneto@icb.ufmg.br 


\section{Abstract}

22 Complex networks have been successfully applied to the characterization and modeling of 23 complex systems in several distinct areas of Biological Sciences. Nevertheless, their utilization in

24 phylogenetic analysis still needs to be widely tested, using different molecular data sets and 25 taxonomic groups, and, also, by comparing complex networks approach to current methods in 26 phylogenetic analysis. In this work, we compare all the four main methods of phylogenetic analysis

27 (distance, maximum parsimony, maximum likelihood, and Bayesian) with a complex networks 28 method that has been used to provide a phylogenetic classification based on a large number of 29 protein sequences as those related to the chitin metabolic pathway and ATP-synthase subunits. In 30 order to perform a close comparison to these methods, we selected Basidiomycota fungi as the 31 taxonomic group and used a high-quality, manually curated and characterized database of chitin 32 synthase sequences. This enzymatic protein plays a key role in the synthesis of one of the exclusive 33 features of the fungal cell wall: the presence of chitin. The communities (modules) detected by the 34 complex network method corresponded exactly to the groups retrieved by the phylogenetic 35 inference methods. Additionally, we propose a bootstrap method for the complex network 36 approach. The statistical results we have obtained with this method were also close to those 37 obtained using traditional bootstrap methods. 


\section{Introduction}

The complex networks approach has been successfully applied to uncover organizational principles underlying the origin, evolution, and functioning of several and distinct complex systems in all areas of science, particularly, in the Biological Sciences (Barabási; Oltvai, 2004).

Among the many developed approaches, we call the attention to the phylogenetic analysis based on complex networks using protein or gene similarity as the only source of information. It precludes the use of any previously developed or newly proposed classification model, as required in currently used methods of phylogenetic analysis (distance, maximum parsimony, maximum likelihood, and Bayesian). The basic ideas and essential tools of this approach were presented and thoroughly discussed in Goés-Neto et al. (2010) and Andrade et al. (2011). Data of four enzymes present in the chitin metabolic pathway for 1695 organisms (with complete genomes) were collected and used to test and validate the method. This framework was also successfully applied to investigate the evolutionary origins of mitochondria using three ATP synthase subunits and its alphaproteobacterial homologs (Carvalho et al., 2015), and to present an evolutionary study of apolipoprotein-E carrying organisms (Benevides et al., 2016). Despite these advances, with large databases, we understand this approach should be widely tested, using distinct databases and taxonomic groups, and comparing its results with those provided the current methods quoted above. In order to confer confidence to such a comparison, it is reasonable to use a high-quality database of sequences related to a small number of organisms as well as to propose a bootstrap procedure for the complex network method.

Nowadays, the taxonomy of Basidiomycota fungi is still strongly based in phylogenetic analyses of nucleotide sequences of rRNA genes (18S, 28S) and spacers (ITS) of nuclear ribosomal DNA as well as some protein-coding genes of nuclear origin (rpb1, rpb2, tefl- $\alpha$ ) (Zhao et al., 
63 2017). However, among the protein-coding genes or their protein products used in phylogenetic

64 reconstructions, none is directly related to exclusive metabolic pathways of fungi, such as that of

65 the biosynthesis of chitin (Pirovani et al, 2005). Therefore, phylogenetic inferences based on

66 unique functional fungal proteins are highly desirable.

Chitin, the linear homopolymer of $\beta-1,4-\mathrm{N}$-acetylglucosamine, is an endogenous structural carbohydrate and one of the main components of the fungal cell wall (Souza et al, 2009). In fungi, chitin is synthesized by a pathway containing six steps and the last and irreversible step corresponds to the conversion of UDP-GlcNAc to chitin by the enzyme chitin synthase (E.C. 2.1.4.16) (Góes-Neto et al., 2010).

In this study, we conduct further investigations on the reliability of the complex network approach addressing two important issues: i) we present a direct comparison of its results to those provided by the four phylogenetic methods indicated before; ii) we propose a bootstrap method that provides a quantitative measure of support values to the branching processes. Although some comparative analysis to other the results of other methods had already been provided, a systematic investigation was missing. On the other hand, a quantitative support for the phylogenetic branches and characterized database of chitin synthases of Basidiomycota fungi from representative species.

\section{Methods}

82 Database

83 The database CHSBasidio was built by data mining using primarily text-based querying in

84 GenPept (NCBI). It comprises complete chitin synthases sequences of Basidiomycota fungi from 
85 model species. Each individual enzymatic protein sequence was stored in a single file containing

86 the protein sequence itself and all the relevant associated information, such as indexers, molecular

87 source, structural and functional information, and complete taxonomic classification of the

88 organism from which the sequence was derived, using automatic procedures specifically

89 developed for these tasks.

90 The knowledge discovery process (KDP) in the CHSBasidio database comprised four

91 steps: (i) data gathering, (ii) screening of collected data, (iii) classification of screened data, and

92 (iv) thorough analyses of classified screened data (Góes-Neto et al., 2010). The gathered data were

93 thus screened to eliminate spurious and doubtful (hypothetical or uncertain) reads, and then

94 organized in structured tables with all the relevant associated information.

95

\section{Molecular Characterization of the CHSBasidio sequences}

The complete sequences of the CHSBasidio database were quali-quantitatively metaanalyzed for identification, characterization and comparison at the protein level. The accessions were analyzed, compared and classified mostly using UniProt (The UniProt Consortium, 2017), according to the following features: (a) sequence length; (b) theoretical molecular mass; (c) theoretical isoelectric point $(\mathrm{p} I)$; (d) transmembrane topological organization (transmembrane regions) using TMHMM v.2.0 (Krogh et al, 2001); (e) conserved domains (Marchler-Bauer et al., 2017); (f) CHS classes (in accordance with the most comprehensive classification recently

104 proposed by Gonçalves et al., 2016).

In order to reveal biological patterns in our CHS database and correlate them to the phylogenetic and complex network analyses, we performed a series of univariate, bivariate and multivariate statistical techniques on this customized database. The statistical description of the 
108 quantitative univariate protein data (number of entries; smallest, largest and mean values of

109 sequence length, molecular mass and isoelectric point; standard error of the estimate of the mean;

110 variance; standard deviation; median; skewness; and kurtosis), as well as correlation (quantitative

111 data) or association (qualitative data) between all the variables (bivariate analyses) were also

112 performed. A multivariate exploratory method to reveal variation trends (ordination) was also

113 carried out for the complete protein sequences data set. The ordination method of Principal

114 Coordinates Analysis (PCOa) was performed using the Gower index as resemblance measure for

115 the mixed qualitative and quantitative variables. All the aforementioned statistical analyses were

116 performed in PAST 3.0 (Hammer; Harper, 2001).

117

\section{Phylogenetic Analyses}

119 The data matrix consisted of 42 sequences from 11 species of basidiomycotan fungi. The 120 sequences were aligned in the most recent version of TCoffee (Notredame et al., 2000), which 121 aligns proteins by combining the output of many aligners as

122 MAFFT/MUSCLE/PROBCONS/POA/DIALIGN/CLUSTALW/PCMA (http://tcoffee.crg.cat/).

123 The gaps were considered as a $21^{\text {st }}$ character state. Phylogenetic analyses were performed in PAUP

124 4.0b10 (Swofford, 2002), and Mr. Bayes 3.2 (Ronquist and Huelsenbeck, 2003), using distance-

125 based (distance matrix) and character-based (maximum parsimony, maximum likelihood and 126 Bayesian) methods.

127 Mean distances and a neighbor-joining algorithm were used for distance analysis, 128 unweighted parsimony for maximum parsimony analysis. VT (Müller; Vingron, 2000) + I

129 (invariable sites) + G (rate heterogeneity among sites: gamma-distributed) best-fit model of protein 130 evolution, previously selected after ProtTest 3.2 (Abascal et al., 2005), was used for maximum 
131 likelihood and Bayesian analyses. Three independent runs were conducted (each with four chains)

132 for $1 \times 10^{6}$ generations for Bayesian inference.

133 Clade robustness was assessed using bootstrap proportions (1,000 replicates) for distance,

134 maximum parsimony, and likelihood analyses, and posterior probabilities proportions for Bayesian

135 analysis. Unrooted trees were edited using Geneious v.9. The resulting single distance and

136 maximum likelihood trees and the majority consensus trees of maximum parsimony and Bayesian

137 inference were then subsequently analyzed.

\section{Network construction and analyses}

All networks in this study were constructed based on the similarity degree between amino acid sequences in proteins of the 42 selected organisms. Whenever necessary, one or more of the

142 following indices, which characterize geometrical and topological aspects a given network, were

143 evaluated (Albert and Barabási, 2002): node degree $\left(k_{i}\right)$, node clustering coefficient $\left(c_{i}\right)$, shortest

144 path between two nodes $\left(d_{i j}\right)$, node betweenness $\left(b^{n}\right)$, edge betweenness $\left(b^{n}{ }_{i j}\right)$, degree assortativity

$145\left(q_{i}\right)$. These indices have local character, as they reflect properties of a given node, of its immediate

146 neighborhood, or of links that are attached to it. Information on the global aspects of the network

147 can be provided by averages of these indices: average degree $(<k>)$, network clustering

148 coefficient $(C)$, average shortest path $(<d>)$, average node betweenness $\left(B_{n}\right)$, average edge

149 betweenness $\left(B_{e}\right)$, average degree assortativity $(Q)$. Other global properties of relevance that have

150 no local counterpart can also be evaluated: network diameter $(D)$, probability distribution of nodes

151 with $k$ links $(p(k))$, probability distribution of node clustering coefficients to node degree $k(C(k))$,

152 fractal dimension $\left(d_{b}\right)$, modularity $\left(m_{d}\right)$. A brief description of the meaning of these indices and

153 functions is provided on the Table 1. 

phylogenetic networks (Bryant \& Moulton, 2004), our method is, in fact, a method to detect communities in generally weighted complex networks (Andrade et al., 2011). When applied to protein similarity networks, it leads to the phylogenetic classification for the organisms associated to the protein sequences, using less biological assumptions than phylogenetic tree and phylogenetic network methods. The method requires the evaluation of some of these indices, particularly $k_{i}, d_{i j}$, and $b^{n}{ }_{i j}$. The whole process can be described in the following steps (1-8):

161 1. Construction of similarity matrices: the $n \times n$ similarity matrix $(S)$, where $n$ indicates the number

162 of organisms, is set up by the comparison of their protein sequences using the most recent version

163 of BLAST (Altschul et al. 1997). The similarity matrix $(S)$ was generated by pairwise alignment, 164 using the score matrix BLOSUM62, which assigns a score for aligning pairs of residues, and 165 determines overall alignment score. The cost to create and extend a gap in alignment is 11 and 1 , 166 respectively. The matrix adjustment method that was used to compensate for amino acid 167 composition of sequences was the conditional composition score matrix adjustment (Altschul et al. 2005). The elements $S_{i j}$ of the similarity matrix $(S)$ are real numbers in the interval $[0,100]$ corresponding to the percentage of agreement of amino acid types between the two sequences. As BLAST may assign a value $S_{j i}$ different from $S_{i j}$, the matrix $S$ is subsequently symmetrized with

171 its elements being defined by $S_{i j}=\min \left(S_{i j}, S_{j i}\right)$. Similarity Networks (PSN) are generated associated with values of a similarity threshold $(\sigma)$

174 according to the following rules: (i) any organism (sequence) is associated to a network node; (ii)

175 a connection is introduced between any pair of nodes $(i, j)$ provided $S_{i j} \geq \sigma$. This criterion based on $176 \sigma$ reflects the evolutionary relationships between the organisms containing the proteins. This 
177 strategy makes it possible to replace one single weighted network defined in terms of $\sigma$ by a family

178 of unweighted networks, which can be analyzed by many developed methods and measures

179 (Albert; Barabási, 2002; Newman, 2003; Boccaletti et al., 2006; Costa et al., 2007).

180 3. Construction of adjacency matrices: any PSN is represented by its adjacency matrix $(M)$,

181 with elements $m_{i j}$ such that: (i) $m_{i j}=1$, if there is a link between nodes $i$ and $j$; (ii) $m_{i j}=0$ otherwise.

182 In the current study we generate a set of 101 networks, one for each integer value of the similarity

183 threshold $\sigma \in[0,100]$.

184

4. Construction of neighborhood matrices $\underline{\hat{M}}$ : for each adjacency matrix $M$, we constructed

185 the set of all neighborhood matrices $M(\square)$ of order $\square=1, \ldots, D$ (Andrade et al., 2006). Any element

$186(M(\square))_{i j}$ is such that it assumes the value 1 only if the shortest path between nodes $i$ and $j$ is $\square$.

187 Otherwise, $(M(\square))_{i j}=0$. Based on the set of $M(\square)$, a neighborhood matrix $\hat{M}$ is constructed 188 according to:

5. Distance between networks: Based on the set of 101 neighborhood matrices, 100 values

191 of the network distance $\delta(\sigma, \sigma+\Delta \sigma)$ between two subsequent networks are evaluated. The network

192 distance $\delta(\alpha, \beta)$ (Andrade et al., 2008) is a measure of how two networks $\alpha$ and $\beta$ are distinct from

193 each other. Here, $\delta(\sigma, \sigma+\Delta \sigma)$ indicates the network distance between two PSN evaluated at nearby

194 values $\sigma$ and $\sigma+\Delta \sigma$, with $\Delta \sigma=1$.

6. Identification of an optimal value of $\sigma$ using the distances $\delta$ : the set of values $\delta(\sigma, \sigma+\Delta \sigma)$

196 is characterized by one or more sharp peaks, indicating the threshold values where important

197 changes in the topological structure of the network occur. The value of $\sigma$ where the maximal value 198 of $\delta(\sigma, \sigma+\Delta \sigma)$ occurs defines the critical network and the critical value $\sigma_{c}$ (Andrade et al., 2008). 
199 Analyses of the properties of the critical network and of the networks close to $\sigma_{c}$, as well as those

200 close to the high peaks, are likely to reveal properties related to an evolutionary branching process.

201 7. Characterization of the properties of the critical network: The network indices indicated

202 in beginning of this subsection are evaluated for the networks indicated before, providing auxiliary

203 information on the community structure of the corresponding networks.

204 8. Identification of communities in critical networks: the final step leading to the separation

205 of organisms into phylogenetic groups corresponds to the detection of modules in the networks

206 selected in the step 6. A module is a subset of nodes such that the number of connections between

207 nodes belonging to the module is proportionally larger than the number of connections between

208 nodes within it to nodes outside it. The Newman-Girvan algorithm (NGA) is used to find the

209 modular structure of the selected networks. It is a bond removal procedure, which amounts to

210 successively removing bonds with largest edge betweenness. At the end of the procedure all bonds

211 are removed, and the community structure analysis results from the dendrogram and the color

212 representation of the renumbered neighborhood matrix (Andrade et al., 2008; Newman \& Girvan, 213 2004).

215 Open Software

We developed a software (still at beta version) to evaluate all necessary steps of the

217 community detection procedure according to the complex network method explained in this paper,

218 which is available for download at http://projectsn.github.io/scannet/. The input data is the

219 similarity matrix which, in the current work, was obtained using BLAST. All results reported in

220 the work that are related to this method were obtained using this software. This does not include

221 the steps related to the evaluation of the bootstrap support measures. 


\section{Comparison of complex network method with tree-based phylogenetic methods}

224 The comparison of complex network with tree-based methods of phylogenetic inference

225 for the same dataset and taxonomic group was carried out by: (i) the congruence index $(G(\varphi, \psi)=$

$226 Q(\varphi, \psi) / R(\varphi, \psi)$, where $R(\varphi, \psi)$ : number of common organisms in the critical networks $\varphi$ and $\psi$ and

$227 Q(\varphi, \psi)$ : number of organisms in the same community in $\varphi$ and $\psi$ (Andrade et al., 2009); (ii)

228 topological comparison of the dendrograms generated by each method; (iii) the corresponding

229 metrics (support indexes) - bootstrap (BS) for distance, maximum parsimony, maximum

230 likelihood, and posterior probability (PP) for Bayesian inference; and (iv) the number of removed

231 edges based on the criterion of largest betweenness degree in the complex networks method.

232 Randomization tests were performed in order to evaluate if there was statistically

233 significant association between the following pair of variables: (i) number of removed edges with

234 largest betweenness degree within the NG procedure and branch length, (ii) number of removed 235 edges during the calculation of betweenness and support indexes of the retrieved groups (BP and 236 PP), using the following parameters: (a) coherence coefficient between variables (resemblance 237 measure) as test criterion ( $\lambda$ ); (b) 1000 iterations, (c) 5\% significance level $(\alpha=0.05)$. The 238 randomization tests were carried out in MULTIV (Pillar, 2001).

241 A bootstrap method for the complex network approach

The bootstrap samples for the complex network method were generated as replicates of the

244 similarity matrix $S$ (see step 1 in section "Network construction and analyses"). In order to generate 
245 a bootstrap sample, each similarity value $S_{i j}$ of the original similarity matrix was divided by 100

246 and taken as the probability $p$ of success in a binomial distribution. Then, 4,944 samples (this

247 number is the size of the overall alignment for the 42 sequences in our study, including gaps) are

248 drawn at random with replacement from this binomial distribution, resulting in $k$ successes.

249 Finally, the corresponding entry $S_{i j}^{\prime}$ in the bootstrap similarity matrix $S^{\prime}$ is obtained by normalizing

250 the number of successes into a percentile score according to the formula:

$251 S_{i j}^{\prime}=k \times 100 / 4944$. Each of the 1,000 similarity matrix bootstrap samples thus generated was then

252 run through the same network-community detection algorithm as the original similarity matrix.

253 The same critical value of $\sigma_{c}$ as the original similarity matrix (for this study, $\sigma_{c}=46 \%$ ) was used

254 for this step. The bootstrap score for any given branch indicates the percentage of bootstrap

255 samples, which featured the community that corresponds to that branch.

\section{RESULTS AND DISCUSSION}

\section{Biological characterization of CHS database}

Our manually curated database comprises 42 unique complete chitin synthase sequences of representative model taxa of the three subphyla of Basidiomycota (Agaricomycotina,

261 Pucciniomycotina, and Ustilaginomycotina), including distinct species with agricultural (the 262 phytopathogens Moniliophthora perniciosa, Puccinia graminis, and Ustilago maydis; and the 263 edible macrofungi, Agaricus bisporus, Flammulina veluticeps, Lentinula edodes, and Pleurotus 264 ostreatus) or medical importance (Filobasidiella neoformans and Malassezia pachydermatis), 265 besides the model species of this phylum (Coprinopsis cinerea) (Table 2).

266 Most of the unique complete protein sequences (76.2\%) in the CHSBasidio database 267 comprise proteins that are either predicted (by in silico translation) or inferred by homology (when 
268 clear orthologs exist in closely related species). Although there are some entries (23.8\%) with

269 evidence at the transcript level, with expression data such as the existence of experimentally

270 produced complete cDNA sequences, there is no accession with clear experimental evidence for

271 the existence of the protein by X-ray structure. This can be reasonably explained by the fact that

272 basidiomycotan chitin synthases have few to many transmembrane regions and occur in the cell

273 membrane. These characteristics make the protein crystallization process difficult.

274 The lengths of the protein sequences range from 519 to 2,066 amino acids residues (mean $275=1,135 \pm 367.68)$, but most of the sequences $(68 \%)$ are in the range from 864 to 1,271 amino 276 acids, exhibiting a marked positive skewness kurtosis. The same is true for the calculated 277 molecular masses of the CHS sequences, which vary from 58.518 to $227.286 \mathrm{KDa}$ (mean = $278128.317 \pm 45.26$ ), since protein length and molecular mass are highly correlated and precisely 279 linear. The calculated isoelectric points $(\mathrm{p} I)$ for the enzymes range from 5.42 to 9.30 (mean $=7.72$ $280 \pm 1.13)$, but most of the enzymes (71\%) are basic. Thus, conversely to protein length and mass, 281 isoelectric point frequency distribution has a marked negative skewness and kurtosis. In fact, both 282 protein length and molecular mass are negatively correlated to isoelectric point, since usually the 283 shorter and lighter the enzymes, the more basic they are (Table 2).

284 Seven distinct patterns of transmembrane topological organization were identified in 285 basidiomycotan chitin synthases and were named profiles 1-7 (1, 2, 3, 4, 5, 6, 7) (Suppl. Figure 1). 286 Profile 2 was the most common (52.4\%), followed by profile $1(26.2 \%)$. These two profiles 287 accounted for most of the entries (78.6\%). Profile 3 occurred in the minority of the accessions, and 288 the four others were unique: each one occurred for only one sequence. Profile 1 comprised six 289 transmembrane helices distributed as followed: two in the N-terminal region, one in the medial 290 region, and three in the C-terminal region of the protein; Profile 2 encompassed seven 
291 transmembrane helices in the C-terminal region; Profile 3 were represented by six transmembrane

292 helices, one in the medial, one in sub-terminal and three other in the C-terminal part of the protein.

293 Profile 4 resembled to some degree the profile 1, as well as profiles 5 and 6 did regarding to profile

294 2. Profile 7 was the most divergent, exhibiting six transmembrane helices, all in the medial region 295 of the protein (Table 2).

296 Three distinct patterns of types of conserved domains were identified in the CHSBasidio 297 database and were designated as profiles 1,2, and 3. Profile 1 corresponded to the sole presence 298 of the conserved domain PF03142. Profile 2 comprised those entries with the conserved domains 299 PF01644 and PF08407. Profile 3 included the accessions containing the conserved domains 300 PF03142 and PF00063. Profile 2 was the most frequent in the sequences (57.1\%). Profile 1 301 appeared in $31 \%$ of the entries while profile 3 was found in only $11.9 \%$ of the complete CHS 302 sequences (Table 2). Furthermore, there is a statistically significant ( $p=1.5016$ E-7) correlation 303 (Spearman coefficient $=0.71)$ between identified transmembrane topological organization and 304 conserved domains profiles. This probably occurred because some of the conserved domains also 305 include transmembrane helices.

306 There are many distinct and, mainly, contradictory classifications of chitin synthase (CHS) 307 isoenzymes in different fungal groups. Recently, Gonçalves et al. (2016) performed a genome308 wide analysis in more than 800 putative chitin synthases in proteomes associated with about 130 309 complete genomes of all known evolutionary lineages of organisms. This large-scale analysis not 310 only allowed the authors to completely revise and unify the fungal CHS classification but also to 311 develop an excellent searchable, web-based reference database

312 (http://wwwabi.snv.jussieu.fr/public/CHSdb/). 
$314 \mathrm{IVb}, \mathrm{Va}, \mathrm{Vb}$. The classes $\mathrm{B}, \mathrm{C}$ and $\mathrm{E}$ are some subdivisions of the classical class II in the older

315 fungal CHS classifications (Nino-Vega et al., 2004). All these aforementioned classes for

316 basidiomycotan CHS, according to Gonçalves et al., 2016, were represented in our CHSBasidio 317 database.

318 Principal Coordinates Analysis (PCoA) was based on five variables (sequence length, 319 isoelectric point, transmembrane topological organization profile, conserved domains profile, and 320 CHS class according to Gonçalves et al., 2016) for the 42 sampling units (entries). The 321 basidiomycotan CHS sequences were ordinated based on the first two coordinates (coord. 1: $32238.23 \%$ and coord. 2: 27.69\%), which jointly represented approximately $66 \%$ of the explained 323 variation according to their qualiquantitative attributes. All classes $\mathrm{Va}$ and $\mathrm{Vb} \mathrm{CHS}$ sequences 324 located in the positive portion of the coordinate 1 and mostly above this coordinate. All classes

325 IVa and IVb CHS sequences occurred in the negative portion of the Coordinate 2 and mostly, in 326 the negative portion of coordinate 1. Conversely, all CHS classes III, B, C and E located in the 327 positive portion of coordinate 2 , with class III sequences in the less positive portion and $\mathrm{B}, \mathrm{C}$, and 328 E in the more positive portion of this coordinate 2 (Fig. 1). Thus, PCO feature-based approach 329 analysis is in complete accordance with the most detailed and large-scale classification of chitin 330 synthases of all fungi until date (Gonçalves et al., 2016).

\section{Tree-based phylogenetic methods}


337 long (including gaps), of which $63 \%$ were variable and $58.4 \%$ of the variable characters were

338 parsimony informative. The final result of the parsimony analysis comprised only one most

339 parsimonious unrooted tree, which showed three distinct clades (named A, B and C), besides a

340 more inclusive clade composed by the union of A and B clades (named AB) (Fig. 2). The first

341 dichotomous division was observed between the most inclusive clade AB and clade C. The most

342 inclusive clade AB showed a 100\% bootstrap value and, thus, it was maximally supported while

343 for clade $\mathrm{C}$ a low support value of only $50 \%$ bootstrap was obtained. Clades A and B, despite being

344 topologically supported by bootstrap values above $50 \%$, showed very contrasting values: clade B,

345 similarly to the most inclusive clade AB, exhibited $100 \%$ bootstrap, while for clade A, topological

346 confidence was a few above half (54\%).

347 Distance

The final result of distance analysis comprised only one unrooted tree. The unrooted tree

349 showed, as well as in parsimony analysis, the same distinct three groups (named as A, B and C),

350 besides a more inclusive group formed by the union of groups A and B (named as AB) (Fig. 2).

351 The most inclusive group AB exhibited, similarly to maximum parsimony analysis, maximum

352 support index, with a 100\% bootstrap value, while group C also had low support (<50\% bootstrap).

353 Both groups A and B had also maximum values of support, with $100 \%$ bootstrap. Conversely to

354 what was retrieved in maximum parsimony analysis, the topological confidence of group A was 355 significantly higher. 
358 distance analyses: the same three distinct groups (named A, B and C), besides a more inclusive

359 group composed by the union of $A$ and $B$ (named as AB) (Fig. 2). The most inclusive group AB

360 showed, similarly to maximum parsimony and distance analysis, the maximum value of bootstrap

361 (100\%), but, conversely to those analyses, group C, as well group AB, also exhibited maximum

362 support value (bootstrap $=100 \%$ ). Group B, individually, also showed a value next to maximum

363 support but slightly lower (97\% bootstrap) than those found in maximum parsimony and distance

364 analyses. Nevertheless, in marked contrast to those analyses, group A had a low support value $(<$

$36550 \%$ bootstrap).

366 Bayesian

The final result of Bayesian analysis was a majority consensus unrooted tree. Once again,

368 it was retrieved the same as obtained in the analysis of maximum parsimony, distance and

369 maximum likelihood, with the same three distinct groups (named as A, B and C), besides the more

370 inclusive group formed by the union of groups A and B (named as AB) (Fig. 2). The most inclusive

371 group $\mathrm{AB}$ showed, similarly to the maximum parsimony, distance and maximum likelihood

372 analyses, the maximum support value (100\% posterior probability), but, conversely to the results

373 obtained in maximum likelihood and similarly to those obtained in maximum parsimony and

374 distance analyses, group C exhibited a low support value (posterior probability $<50 \%$ ). Group B,

375 as in maximum parsimony and distance analyses, also exhibited the maximum support value,

$376100 \%$ posterior probability. Nevertheless, similarly to distance analysis and differently from

377 maximum parsimony and maximum likelihood, group A had a significantly high support, with $37893 \%$ posterior probability. 
Table 3 comparatively summarizes the support indexes of bootstrap (BP) and posterior 380 probability (PP), retrieved from the four tree-based phylogenetic analysis methods for the studied 381 database (non-congruent indexes amongst different methods are in red).

In all the tree-based phylogenetic analyses (distance, maximum parsimony, maximum

383 likelihood and Bayesian), the group A comprised only basidiomycotan CHS isoenzymes of the

384 classes B, C and E, while group B encompassed exclusively CHS of the class III, and group C 385 consisted of all CHS of the classes IVa, IVb, Va and Vb. Moreover, the group AB, retrieved in all 386 phylogenetic analyses corresponded exactly to the join grouping of classes B, C, E and III CHS 387 sequences. Therefore, all the four phylogenetic methods retrieved exactly the fungal CHS 388 classification of Gonçalves et al. (2016) for the Phylum Basidiomycota.

390 Complex Networks method

The optimum value of similarity (critical similarity) used to retrieve the phylogenetic relations (Andrade et al., 2011) was $\sigma_{c}=46 \%$ (Fig. 3). Near this critical value, there is an abrupt topological change with the disaggregation of a sole network completely connected in groups that

394 can be discerned, with maximum phylogenetically-relevant information in relation to noise, 395 enabling the detection of communities (modules) in the network (Fig. 4).

396 The selected critical network that best represented the phylogenetic relationships of the 397 studied dataset has order $(N)=42$ nodes and size $(M)=198$ edges.

398 This critical network has the following indexes in relation to:

399 I. Connectivity: (i) the degrees of nodes varies from 1 to 13 and the average degree is 400 $<k>=9.429$; (ii) the probability distribution of nodes with degree $\mathrm{k}$ corresponds to a bimodal 
401

402

403

404

405

406

407

408

409

410

411

412

413

414

415

416

417

418

419

420

421

422

distribution: 7 nodes with low value of $\mathrm{k}$ centered in $\mathrm{k}=3$, and 35 nodes with high values of $\mathrm{k}$ centered in $\mathrm{k}=11$;

II. Assortativity $\mathrm{Q}=0.00216$

III. Distance: (iv) average minimal path is $\langle d\rangle=3.272$, (v) diameter $D=7$, (vi) average node betweenness $B_{n}=137.4$ (vii) average edge betweenness $B_{e}=3.27$;

IV. Cluster: (viii) average clustering coefficient $C=0.815$; mainly in the interval $[0.6,1.0]$, only 5 nodes with $c_{i}$ value below 0.6 .

V. Auto-similarity: (ix) fractal dimension $d_{b}=1.37$

VI. $\quad$ Modularity $m_{d}=0.975$

The detection of modular structure, that is, the identification of communities in the critical network $\sigma_{\text {crit }}=46 \%$ was carried out by joint analysis of the color representation of the neighborhood matrix (Fig. 5) along with the dendrogram generated by successive link elimination according to the betweenness index (using edge betweenness) (Fig. 6).

The critical network $\sigma_{\text {crit }}=46 \%$ exhibited three very distinct communities (modules), named $\mathrm{C} 1, \mathrm{C} 2$, and $\mathrm{C} 3$ (Fig. 7). The community $\mathrm{C} 2$ was the one with highest connectivity, as one can see in the color matrix.

Communities C2 and C3 showed more connected between them, with four nodes with a high edge betwenness value (C2: 16 and 23; C3: 19 and 29) and four edges with high edge betweenness (inter-communities: 29-23, 29-16; 19-23, 19-16) that connect them. Community C1, however, had only one inter-community edge, linking the vertices $\mathrm{C} 1: 12$ and $\mathrm{C} 3: 38$. 
423 Comparison of the complex networks method and traditional tree-based methods of phylogenetic analyses

The classifications generated by the complex networks method and the four tree-based 426 methods of phylogenetic analysis (distance, maximum parsimony, maximum likelihood, Bayesian) exhibited maximum congruence index: $G(\varphi, \psi)=100 \%$, with $\varphi, \psi$ corresponding to a community $\mathrm{CX}$ generated by any two methods. The communities $\mathrm{C} 1, \mathrm{C} 2$ and $\mathrm{C} 3$, detected by the complex networks method, corresponded exactly to the groups $\mathrm{C}, \mathrm{B}$ and A obtained through the phylogenetic inference methods, respectively, and, thus, the critical network $\left(\sigma_{\text {crit }}=46 \%\right)$ retrieved

431 all the phylogenetic relationships of the basidiomycotan CHS. The closer relationship of groups A 432 and $\mathrm{B}$, forming the more inclusive group $\mathrm{AB}$, was also detected by the complex networks method, 433 through the higher number of vertices connected by inter-community edges of high betweenness 434 between $\mathrm{C} 2$ and C1. Furthermore, additional phylogenetic relationships, which cannot be directly 435 visualized by a tree graph, were also evidenced as exhibited by the intercommunitary edges 436 between nodes in communities $\mathrm{C} 2$ and C3, and between communities $\mathrm{C} 1$ and $\mathrm{C} 3$ (Table 2 and Fig. 437 7).

Table 4 synthesizes, in a comparative manner, the indices of number of removed edges (in 439 the complex networks method) and the support indexes of bootstrap (BP) and posterior probability 440 (PP) (in the maximum parsimony, distance, maximum likelihood, and Bayesian methods) for the 441 main retrieved groups/communities. However, a statistically significant correlation between the 442 number of removed edges by the complex networks method and the support measures of the 443 traditional methods of phylogenetic analysis was not detected. 
445 representation of evolutionary relationships between included organisms or their molecules, such

446 as proteins (Russo et al., 2012). The trees are generated based on the assumption that the

447 evolutionary process is strictly divergent but the widespread existence of reticulated evolution

448 (horizontal/lateral transfers, hybridizations, and non-dicothomous cladogenesis) implies that the

449 evolutionary process can be concurrently divergent and convergent so that the best graphic 450 representation would not be a tree (Kunin et al, 2005). Therefore, a more realistic graphical 451 representation of phylogenetic hypotheses can be generated by a complex networks method 452 (Bapteste et al, 2013). Tree graphs are a subset of general graphs or networks. Trees are optimized 453 visualizations of often much more complex evolutionary signals. Using trees, additional 454 dimensions of information in the data are overlooked (Bapteste et al., 2012). The scope of our 455 evolutionary thinking should be moved beyond a tree-thinking to a network-thinking paradigm 456 (Bapteste et al., 2013).

\section{Comments on the used bootstrap method}

The topologies generated by the phylogenetic analyses must be submitted to some groups (Felsenstein, 2004). The percentage values of bootstrap (distance, maximum parsimony, maximum likelihood, Bayesian) and posterior probability (Bayesian) are the most used support measures: the closer to the maximum value, the more robust is the retrieved topology, that is, the more reliable is the formed group/community. Using the bootstrap method based on random resampling over similarity scores, we have obtained results that were strikingly similar to those used for the tree-based methods. Notably, the same groups A, B and C as in the tree-based methods 
467 were also retrieved by the complex network method, with bootstrap values of $100 \%, 100 \%$, and

$46874.6 \%$, respectively. In this scenario, we also found a bootstrap support of $100 \%$ for group AB 469 (Fig. 8).

470 This bootstrap procedure is distinct from those used in the tree-based methods since it does 471 not rely on the resampling of characters (Felsenstein 2004); instead, the locus of the resampling 472 procedure is here the similarity matrix, which serves as the starting point for the community 473 detection algorithm. The rationale behind this procedure is that it may emulate to some degree the 474 consequences on the similarity matrix that would be obtained if one were to resample characters 475 (i.e., amino acids) directly, since this would plausibly result in a variance on the similarity scores 476 that would depend on the similarity scores themselves. That is, by resampling characters, we could 477 expect a peak of variance in similarity scores across bootstrap samples around the similarity score 478 of $50 \%$, since at this point there would be a greater margin for the alignment of bootstrap sequences 479 to either improve or worsen over the original sequences. We also expect the variance for bootstrap 480 similarity scores to decrease as we approach either end of the similarity spectrum. The method of 481 resampling over a binomial distribution herein adopted emulates this behavior. Across our 482 bootstrap samples, the highest variance (0.66) was found in one of the entries with an original 483 similarity score of 46, while an entry with an original similarity score of 95 exhibited a variance 484 of 0.11 (Suppl. Table 1).

It should be noted that, by performing a bootstrap resampling according to the method here 486 described, each bootstrap sample results in an adjacency matrix $m$ (see section "Network 487 construction and analyses", step 3, above) that is slightly different than the one obtained from the 488 original similarity matrix and $\sigma_{c}$. We also performed tests in which the bootstrap samples were generated by direct random rewiring of the adjacency matrix itself, yielding similar results to the 
490 method adopted herein. However, the rewiring method demands additional statistical assumptions

491 for which we are not ready to provide support at this point.

492 It may be objected that this bootstrap method yields scores that cannot be compared to

493 those in the tree-based methods. Strictly speaking, however, bootstrap scores for the complex

494 network-based method are calculated in exactly the same way as in the other methods; what differs

495 is the resampling method. While traditional resampling from sequences, followed by the 496 application of the network-based method, can in principle be done for each bootstrap sample, in

497 practice this is computationally much more demanding than resampling from the similarity matrix.

498 The degree to which those methods are equivalent will be the subject of a future study. In complex networks, the statistical measure that could be used in a similar manner as the support measures used in phylogenetic analyses (BP and $\mathrm{PP}$ ) is the number of removed edges

501 during the calculation of betweenness. When one removes edges with high betweenness, a great

502 perturbation in the system is caused, which can imply in the rupture of network structure, and the

503 more resilient to attacks a community/group is, the more robust it is (Costa et al. 2007). In this

504 case study with chitin synthases of Basidiomycota fungi, the community C2 (= group B in 505 phylogenetic analyses) was the one that exhibited the highest value when considering this 506 statistical measure. Nonetheless, the lack of statistically significant correlation between a probable 507 support measure for communities in complex networks and the traditionally used support measures 508 in phylogenetic methods (BP and PP) involving a same dataset suggests the need of a more 509 comprehensive investigation about this topic. Currently, our research group has been generating 510 and analyzing many complex networks with high order and size to answer this fundamental 511 question. 


\section{Concluding Remarks}

514 In this work, we compared a complex networks method with the traditional methods of

515 phylogenetic analysis (distance, maximum parsimony, maximum likelihood, and Bayesian), using

516 a manually curated and characterized database of chitin synthases of Basidiomycota fungi from

517 model species. The three modules detected by the complex networks method corresponded exactly

518 to the groups retrieved by the aforementioned phylogenetic inference methods. By applying the

519 method again to the values of $\sigma=52,63$ and 65 , which locate the three small secondary peaks in

520 the Figure 4, we were able to provide a finer sub-community analysis. The intra-community links

521 that survive in the presented analyses correspond to adjacency matrix elements $m_{i j}=1$ and

522 similarity score $S_{i j}>46$, which are about to be erased at these higher threshold values. Furthermore,

523 we proposed and successfully tested, for the first time, a bootstrap method, whose results were

524 close to those obtained using traditional bootstrap in current phylogenetic methods.

Finally, we remark that complex network formalism can be applied to investigation subsequent transitions that give rise to sub-communities within $\mathrm{C} 1, \mathrm{C} 2$ and $\mathrm{C} 3$. To this purpose we use the secondary peaks of $\delta(\sigma, \sigma+\Delta \sigma)$ identified at $\sigma=52,63$, and $65 \%$. Then, the same sequence of steps (2-8) indicated previously at these values $\sigma$ to obtain dendrograms and neighborhood matrices uncovering the subcommunity structure.

\section{Acknowledgements}

We thank all who contributed directly or indirectly to this work, especially the $\mathrm{CNPq}$

533 (Conselho Nacional de Desenvolvimento Científico e Tecnológico) and the Graduate Programs of 
534 Microbiology and Bioinformatics of the Universidade Federal de Minas Gerais (UFMG) for

535 infrastructure and for partially financing this study, and also, Jose Mario Vicensi Grzybowski for

536 help with the computational implementation of the community-detection algorithm.

\section{REFERENCES}

Abascal F, Zardoya R, Posada, D. 2005. ProtTest: Selection of best-fit models of protein evolution. Bioinformatics: 21:2104-2105. DOI: 10.1093/bioinformatics/bti263.

542 Albert R, Barabási AL. 2002. Statistical mechanics of complex networks. Rev

543 Mod Phys 74: 47-97. DOI: 10.1103/RevModPhys.74.47.

544

545 Altschul SF, Madden TL, Schäffer AA, Zhang J, Zhang Z, Miller W, Lipman DJ. 1997. Gapped

BLAST and PSI-BLAST: A new generation of protein database search programs. Nucleic Acids

Res 25: 3389-3402. DOI: 10.1093/nar/25.17.3389.

548

549 Altschul, S.F., Wootton, J.C., Gertz, E.M., Agarwala, R., Morgulis, A., Schaffer, A.A. and Yu, Y.550 K. (2005) "Protein database searches using compositionally adjusted substitution matrices," FEBS J 272(20):5101-9.

552

553 Andrade RFS, Miranda JGV, Lobão TP. 2006. Neighborhood properties of 554 complex networks. Phys Rev E 73: 046101. DOI: 10.1103/PhysRevE.73.046101. 
556 Andrade RFS, Miranda JGV, Pinho STR, Lobão TP. 2008. Measuring distances between complex

557 networks. Phys Lett A 372: 5265-5269. DOI: 10.1016/j.physleta.2008.06.044.

558

559 Andrade RFS, Pinho STR, Lobão TP. 2009. Identification of community structure in networks

560 using higher order neighborhood concepts. Int. J. Bifurc. Chaos. 19: 2677-2685. DOI:

$561 \quad 10.1142 / \mathrm{S} 0218127409024396$.

562

563 Andrade RFS, Rocha-Neto IC, Santos LBL, de Santana CN, Diniz MVC, Lobão, TP, Góes-Neto,

564 A, Pinho, STR, El-Hani, CN. 2011. Detecting Network Communities: An Application to

565 Phylogenetic Analysis. PLoS Comput Biol 7(5): e1001131. DOI: 10.1371/journal.pcbi.1001131.

566 Bapteste E, Lopez P, Bouchard F, Baquero F, McInerney JO, Burian RM. 2012. Evolutionary

567 analyses of non-genealogical bonds produced by introgressive descent. Proc. Natl. Acad. Sci.

568 U.S.A. 109, 18266-18272. DOI: 10.1073/pnas.1206541109.

569

570 Bapteste E, van Iersel L, Janke A, Kelchner S, Kelk S, McInerney JO, Morrison DA, Nakhleh L,

571 Steel M, Stougie L, Whitfield, J. 2013. Networks: expanding evolutionary thinking. Trends in

572 Genetics, 29:439-441. DOI: 10.1016/j.tig.2013.05.007.

573

574 Barabasi AL, Oltvai ZN. 2004. Network biology: understanding the cell's functional organization.

575 Nat. Rev. Genet. 5, 101-113. DOI: 10.1038/nrg1272.

576 
577 Benevides, LJ; Carvalho, DS; Andrade, RFS; Bonfim, GC; Fernandes, FMC 2016. Evolutionary

578 analysis of apolipoprotein E by Maximum Likelihood and complex network methods. Genetics 579 and Molecular Biology 26, 042317. DOI: 10.1590/1678-4685-gmb-2015-0164.

580

581 Boccaletti S, Latora V, Moren Y, Chavez M, Hwang DU. 2006. Complex Networks: structure and 582 dynamics. Phys Rep 424: 175-308. DOI: 10.1016/j.physrep.2005.10.009.

583

584 Bryant D, Moulton V. 2004. Neighbor-net, an agglomerative method for the construction of 585 phylogenetic networks. Mol. Biol. Evol. 21: 255-265. DOI: 10.1093/molbev/msh018

586

587 Costa LF, Rodrigues FA, Travieso G, Villas-Boas PR. 2007. Characterization of complex 588 networks: A survey of measurements. Advances in Physics 56: 167-242. DOI: $10.1080 / 00018730601170527$.

590

591 Carvalho DS, Andrade RFS, Pinho STR, Góes-Neto A, Lobão TCP, Bomfim GC, El-Hani CN.

592 2015. What are the Evolutionary Origins of Mitochondria? A Complex Network Approach. PLoS 593 ONE 10(9): e0134988. DOI: 10.1371/journal.pone.0134988.

595 Felsenstein J. 2004. Inferring Phylogenies. Sunderland, MA: Sinauer Associates. 596

597 Góes-Neto A, Diniz MVC, Santos LB, Pinho ST, Miranda JG, Lobão TP, Borges EP, El-Hani CN, 598 Andrade RFS. 2010. Comparative protein analysis of the chitin metabolic pathway in extant 
599 organisms: A complex network approach. BioSystems 101: 59-66. DOI:

$600 \quad$ 10.1016/j.biosystems.2010.04.006.

601

602 Gonçalves IR, Brouillet S, Soulié MC, Gribaldo S, Sirven C, Charron N, Boccara M, Choquer M.

603 2016. Genome-wide analyses of chitin synthases identify horizontal gene transfers towards 604 bacteria and allow a robust and unifying classification into fungi. BMC Evolutionary 605 Biology, 16(1), p.252. DOI: 10.1186/s12862-016-0815-9.

606

607 Hammer Ø, Harper DAT, Ryan PD. 2001. PAST: Paleontological Statistics Software Package for 608 Education and Data Analysis. Palaeontologia Electronica 4(1): 9pp.

609

610 Karrer B, Levina E, Newman MEJ. 2008. Robustness of community structure in networks. 611 Physical Review E 77: 046119. DOI: 10.1103/PhysRevE.77.046119.

612

613 Kearse, M, Moir, R, Wilson, A. Stones-Havas, S, Cheung, M, Sturrock, S, Buxton, S, Cooper, A, 614 Markowitz, S, Duran, C, Thierer, T, Ashton, B, Meintjes, P. and Drummond, A. (2012) Geneious

615 Basic: an integrated and extendable desktop software platform for the organization and analysis of 616 sequence data. Bioinformatics. 28, 1647-1649. DOI: 10.1093/bioinformatics/bts 199 .

617

618 Krogh A, Larsson B, von Heijne G, Sonnhammer EL. 2001. Predicting transmembrane protein 619 topology with a hidden Markov model: application to complete genomes. J. Mol. Biol. 305, 567620 580. DOI: $10.1006 /$ jmbi.2000.4315.

621 
622 Kunin V, Goldovsky L, Darzentas N, Ouzounis CA. 2005. The net of life: reconstructing the 623 microbial phylogenetic network. Genome Research 15: 954-959. DOI: 10.1101/gr.3666505.

624

625 Letunic I, Bork P. 2011. Interactive Tree Of Life v2: online annotation and display of phylogenetic 626 trees made easy. Nucleic Acids Res DOI: 10.1093/nar/gkr20.

627

628 Marchler-Bauer A, Bo Y, Han L, He J, Lanczycki CJ, Lu S, Chitsaz F, Derbyshire MK, Geer RC, 629 Gonzales NR, Gwadz M, Hurwitz DI, Lu F, Marchler GH, Song JS, Thanki N, Wang Z, Yamashita 630 RA, Zhang D, Zheng C, Geer LY, Bryant SH. 2017. CDD/SPARCLE: functional classification of 631 proteins via subfamily domain architectures. Nucleic Acids Res. 4,45(D1): D200-D203. DOI: $63210.1093 /$ nar/gkw1129.

633

634 Muller T, Vingron M. 2000. Modeling amino acid replacement. J Comput Biol 7:761-776. DOI: $63510.1089 / 10665270050514918$.

636

637 Newman MEJ. 2003. The Structure and Function of Complex Networks. SIAM

638 Review 45: 167-256. DOI: 10.1137/S003614450342480.

639

640 Newman, MEJ. 2004. Fast algorithm for detecting community structure in

641 networks. Phys Rev E 69: 066133. DOI: 10.1103/PhysRevE.69.066133.

642

643 Newman MEJ, Girvan M. 2004. Finding and evaluating community structure in networks. 644 Physical Review E 69: 026113. DOI: 10.1103/PhysRevE.69.026113. 
646 Niño-vega GA, Carrero L, San-Blas G. 2004. Isolation of the CHS4 gene of Paracoccidioides

647 brasiliensis and its accommodation in a new class of chitin synthases. Med Mycol, 42: 51-7 DOI:

$648 \quad 10.1080 / 1369378031000153811$

649

650 Notredame C, Higgins DG, Heringa J. 2000. T-Coffee: A novel method for fast and accurate 651 multiple sequence alignments. J Mol Biol, 302: 205-217. DOI: 10.1006/jmbi.2000.4042.

652

653 Pillar VP. 2001. MULTIV - Multivariate Exploratory Analysis, Randomization Testing and 654 Bootstrap Resampling. Software manual.

655

656 Pirovani CP, Lopes MA, Oliveira BM, Dias CV, Souza CS, Galante RS, Santos-Junior MC, Silva 657 BGM, Uetanabaro APT, Taranto AG, Cruz SH, Roque MRA, Micheli FFL, Gesteira AS, Schriefer 658 A, Cascardo JCM, Pereira GAG, Góes-Neto A, 2005. Knowledge discovery in genome database: 659 the chitinmetabolic pathway in Crinipellis perniciosa. In: Proceedings of IV Brazilian Symposium 660 on Mathematical and Computational Biology/I InternationalSymposium on Mathematical and 661 Computational Biology, vol. 1, E-Papers Serviços Editoriais LTDA, Rio de Janeiro, pp. 122-139. 662

663 Ronquist FR, Huelsenbeck JP. 2003.MrBayes 3: Bayesian phylogenetic, inference under mixed 664 models. Bioinformatics 19:1572-1574. DOI: 10.1093/bioinformatics/btg180.

665

666 Russo CAM, Miyaki CY, Pereira SL. Reconstrução filogenética: Métodos geométricos. In: Matioli 667 SR, editor. Biologia Molecular e Evolução. Ribeirão Preto: Holos Editora; 2012. p. 108-16. 202p. 
668

669 Saitou N, Nei M. 1987. The Neighbor-Jointing Method: a new method for reconstruction of 670 phylogenetic trees. Mol. Biol. Evol. 4:406-425.

671

672 Souza CS, Oliveira BM, Costa GG, Schriefer A, Selbach-Schnadelbach A, Uetanabaro APT, 673 Pirovani, CP, Pereira, GAG, Taranto, AG, Cascardo, JCM, Goes-Neto, A. 2009. Identification and 674 characterization of a class III chitin synthase gene of Moniliophthora perniciosa, the fungus that 675 causes witches' broom disease of cacao. The Journal of Microbiology, 47: 431-440. DOI: $67610.1007 / \mathrm{s} 12275-008-0166-3$.

677

678 Swofford DL. 2002. PAUP phylogenetic analysis using parsimony and other methods, version 679 4.0b10. Sinauer, Sunderland.

680

681 The UniProt Consortium. 2017. UniProt: the universal protein knowledgebase. Nucleic Acids Res. 682 45: D158-D169. DOI: 10.1093/nar/gkw1099.

683

684 Zhao RL, Li GJ, Sánchez-Ramírez S, Stata M, Yang ZL, Wu G, Dai YC, He SH, Cui BK, Zhou 685 JL, Wu F. 2017. A six-gene phylogenetic overview of Basidiomycota and allied phyla with 686 estimated divergence times of higher taxa and a phyloproteomics perspective. Fungal Diversity, 687 pp.1-32. DOI: 10.1007/s13225-017-0381-5.

688

689

FIGURES: 
690 Fig. 1: Principal Coordinates Analysis (PCOa) of the qualiquantitaive feature-based matrix of

691 complete sequences of proteins of CHSBasidio database. The variations explained by the

692 coordinates were: coord. 1 (34.81\%) and coord. $2(21.78 \%)$.

693

694 Fig. 2: Maximum Parsimony majority-rule consensus tree; Distance tree; Maximum Likelihood 695 tree; Bayesian majority-rule consensus tree. (Bootstrap values above 50\% are exhibited. Scale bar 696 represents the number of amino acid substitutions).

697

698 Fig. 3: The distance $\delta(\sigma, \sigma+\Delta \sigma)$ between networks for successive similarities 699 at the maximal value with $\Delta \sigma=1$.

700 Fig. 4: Size of the largest connected component $(\mathrm{Nc})$ versus the threshold similarity $\sigma$.

701 Fig. 5: Colour plot of neighbourhood matrix at $\sigma_{\text {cri }}=46 \%$ with the indication of the communities 702 (modules).

703 Fig. 6: Dendrogram produced by the successive elimination of edges with the largest value of 704 betweeness.

705 Fig. 7: The standard network representation at $\sigma_{\text {cri }}=46 \%$ (Gephi) with the indication of the 706 communities (modules).

707

708 Fig. 8: Dendrogram produced by the successive elimination of edges with the largest value of

709 betweenness. Bootstrap values for all branches were produced according to the method described

710 in the text. Bootstrap support for communities $\mathrm{AB}(\mathrm{C} 3+\mathrm{C} 2), \mathrm{A}(\mathrm{C} 3), \mathrm{B}(\mathrm{C} 2)$, and $\mathrm{C}(\mathrm{C} 1)$ in

711 complex network method. (Note: The terminology A, B and C is those used in tree-based methods). 


\section{TABLES:}

714

715 Table 1: Description of the most usual indices and functions used to characterize a network.

716

717 Table 2: List of the basidiomycotan chitin synthase sequences and their corresponding

718 qualiquantitative features and assignment to the groups retrieved in phylogenetic tree-based and

719 network-based analyses.

720

721 Table 3: Comparison of the support indexes of the tree-based methods.

722

723 Table 4: Comparison of number of removed edges and support indexes of tree-based and complex

724 networks methods.

725

726 SUPPLEMENTARY MATERIAL

727

728 Supplementary Figure 1 (a-g): Patterns of transmembrane topological organization of CHS 729 sequences.

730

731 Supplementary Table 1: Variance of each similarity matrix element calculated from 1,000

732 bootstrap samples. 
Figure 1

Principal Coordinates Analysis (PCOa) of the qualiquantitaive feature-based matrix of complete sequences of proteins of CHSBasidio database.

The basidiomycotan CHS sequences were ordinated based on the first two coordinates (coord. 1: $38.23 \%$ and coord. 2: $27.69 \%$ ), which jointly represented approximately $66 \%$ of the explained variation according to their qualiquantitative attributes.

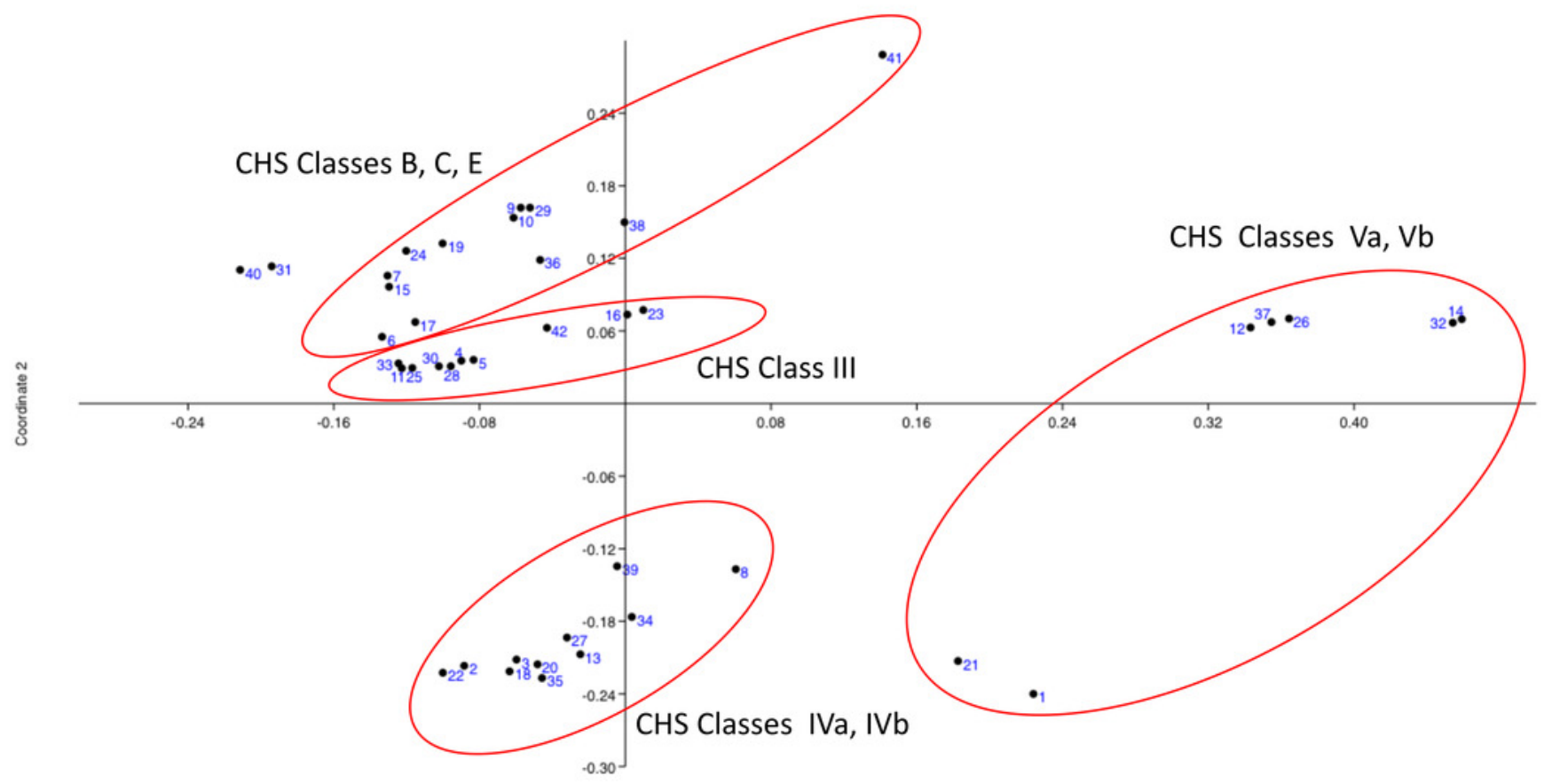

Coordinate 1 
Figure 2

(a) Maximum Parsimony majority-rule consensus tree; (b) Distance tree; (c) Maximum Likelihood tree; (d) Bayesian majority-rule consensus tree.

Trees of the fourtree-based phylogenetic methods. Bootstrap values above $50 \%$ are exhibited. Scale bar represents the number of amino acid substitutions.
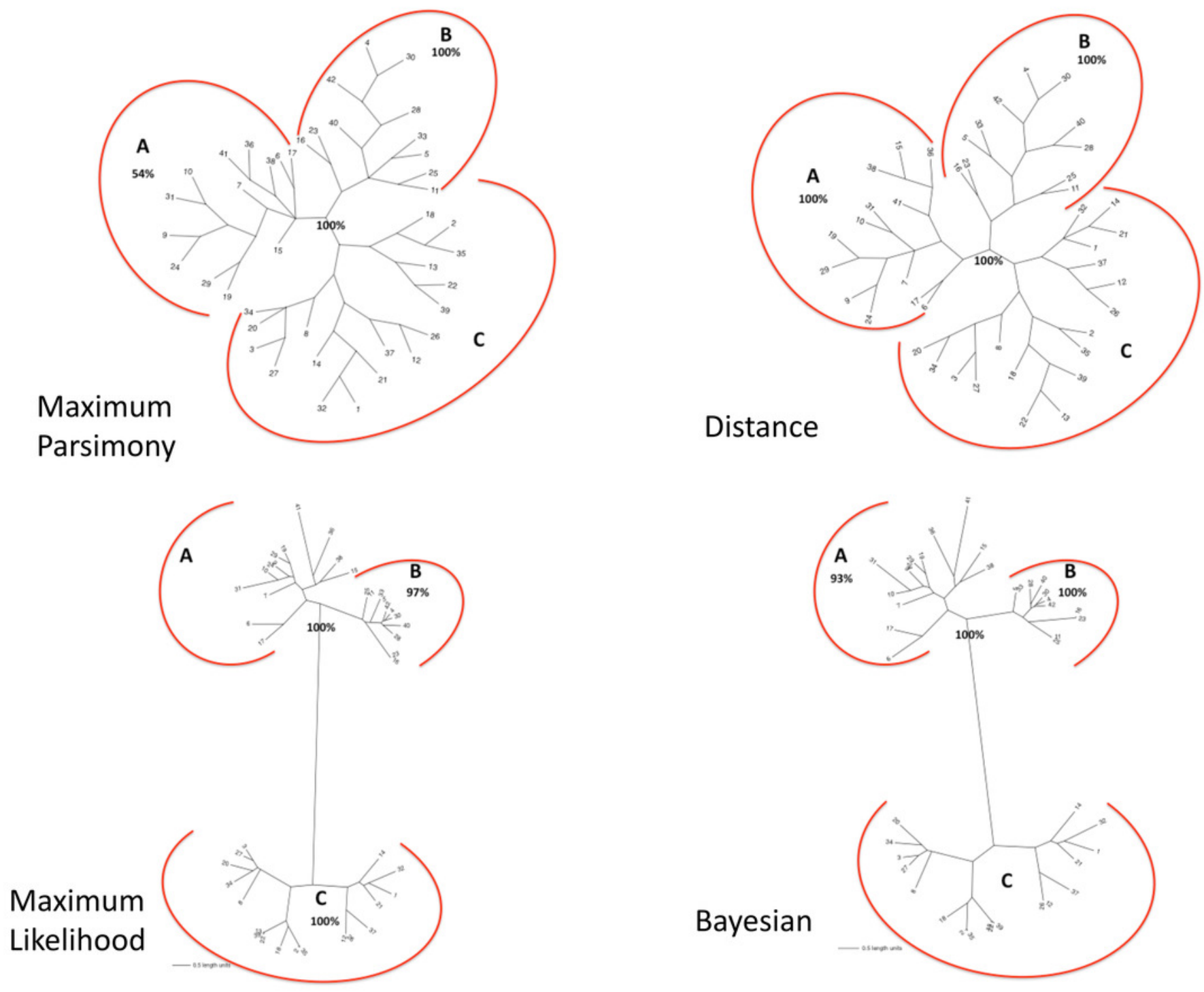
Figure 3

The distance $\delta(\sigma, \sigma+\Delta \sigma)$ between networks for successive similarities at the maximal value with $\Delta \sigma=1$.

The optimum value of similarity (critical similarity) used to retrieve the phylogenetic relations (Andrade et al., 2011).

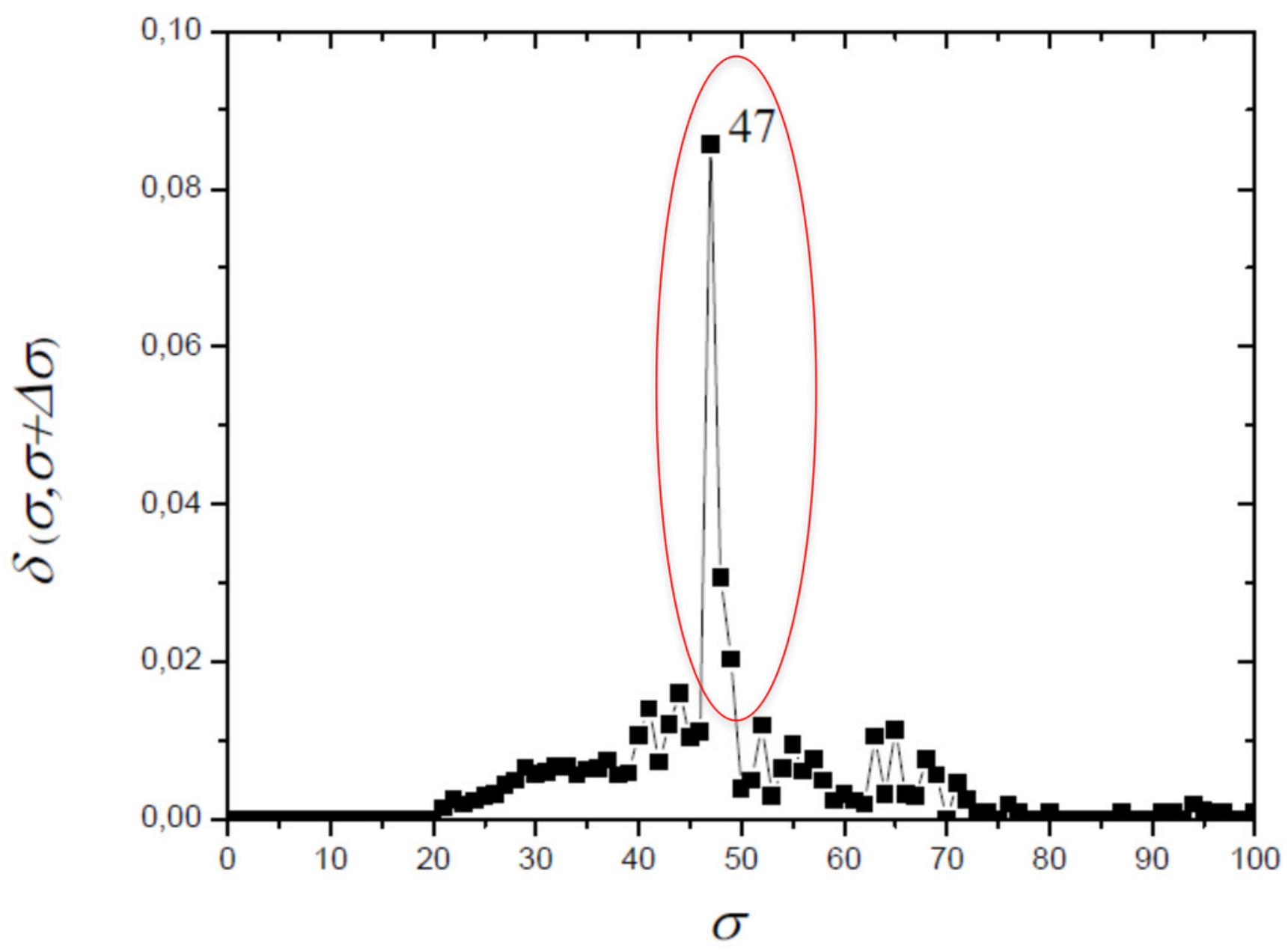




\section{Figure 4}

Size of the largest connected component (Nc) versus the threshold similarity $\sigma$.

Near the critical value, there is an abrupt topological change with the disaggregation of a sole network completely connected in groups that can be discerned, with maximum phylogenetically-relevant information in relation to noise, enabling the detection of communities (modules) in the network.

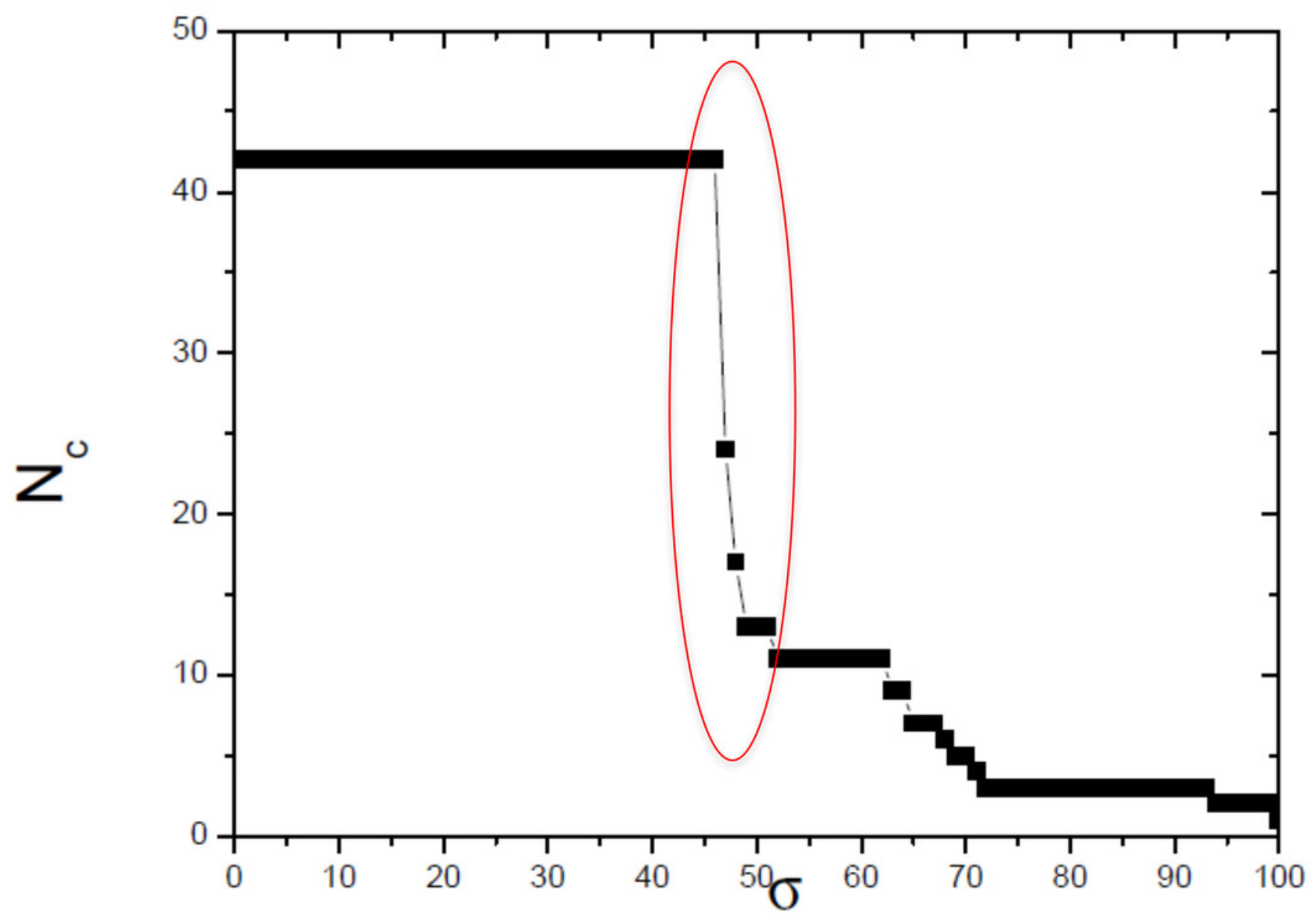




\section{Figure 5}

Colour plot of neighbourhood matrix at $\sigma_{\text {cri }}=46 \%$ with the indication of the communities (modules).

The detection of modular structure, that is, the identification of communities in the critical network was carried out by joint analysis of the color representation of the neighborhood matrix
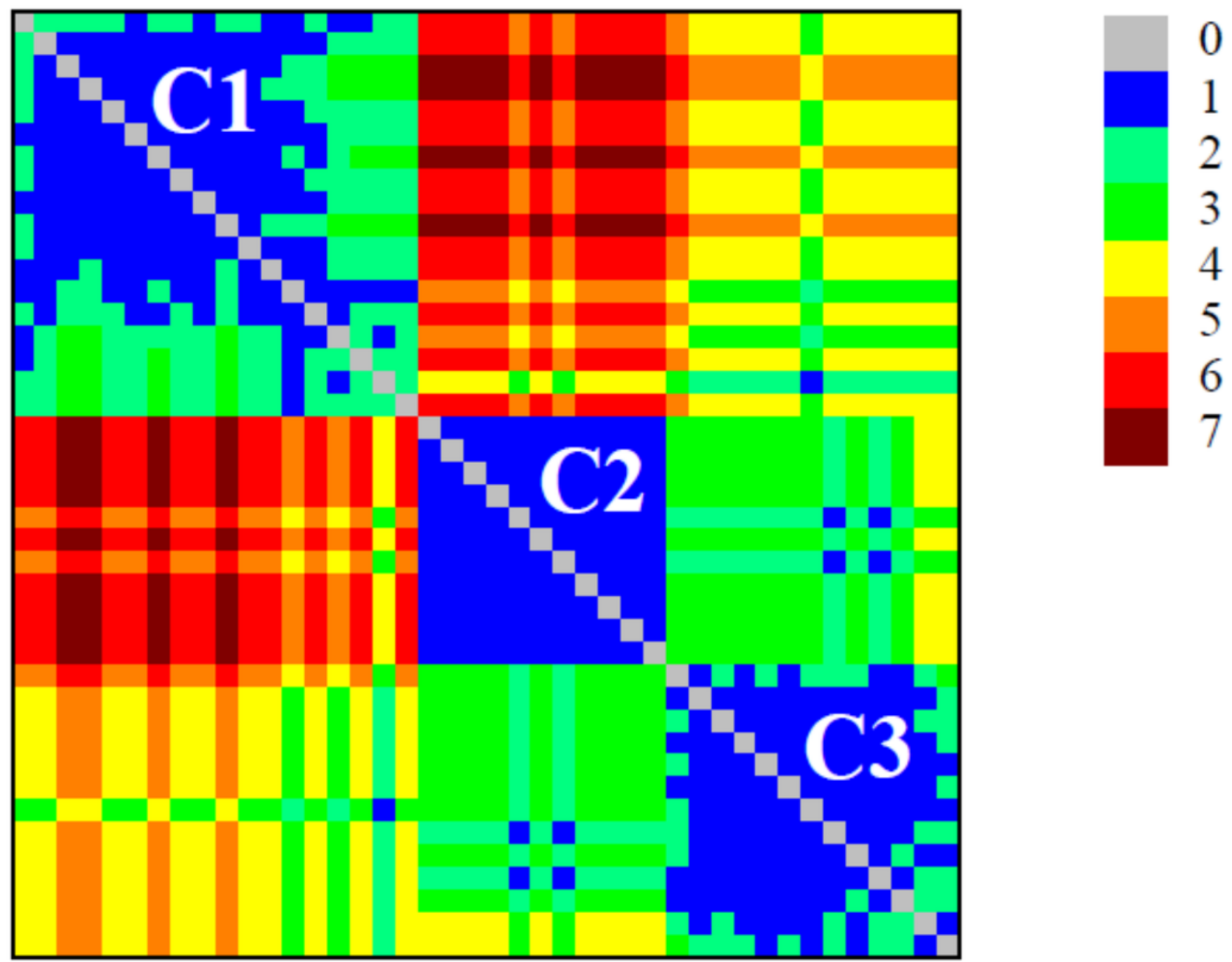
Figure 6

Dendrogram produced by the successive elimination of edges with the largest value of betweenness.

The detection of modular structure, that is, the identification of communities in the critical network was carried out by joint analysis of the color representation of the neighborhood matrix (Fig. 5) along with the dendrogram generated by successive link elimination according to the betweenness index.

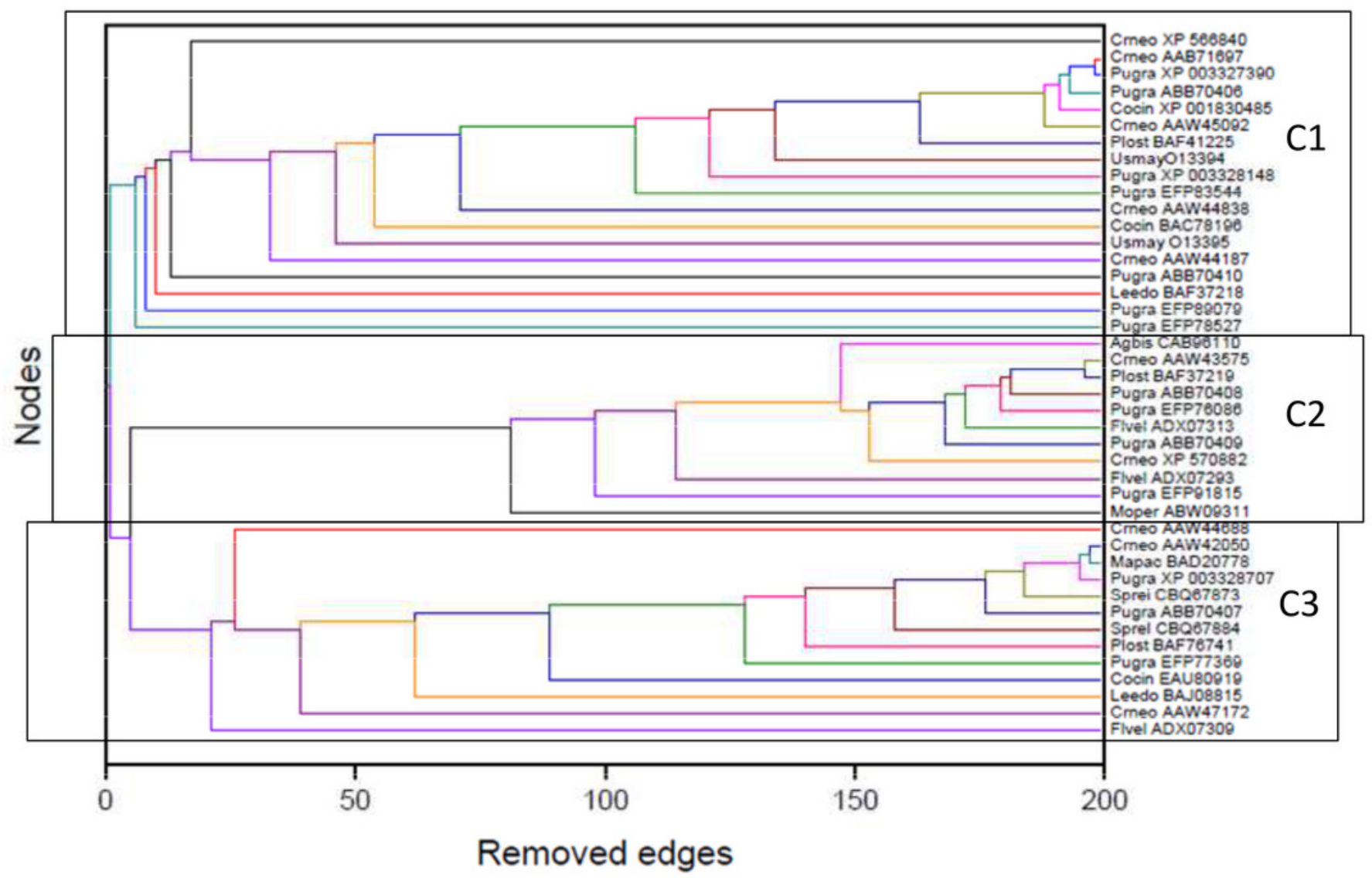




\section{Figure 7}

The standard network representation at $\sigma_{\text {cri }}=46 \%$ (Gephi) with the indication of the communities (modules).

The critical network exhibited three very distinct communities (modules), named $\mathrm{C} 1, \mathrm{C} 2$, and C3. 


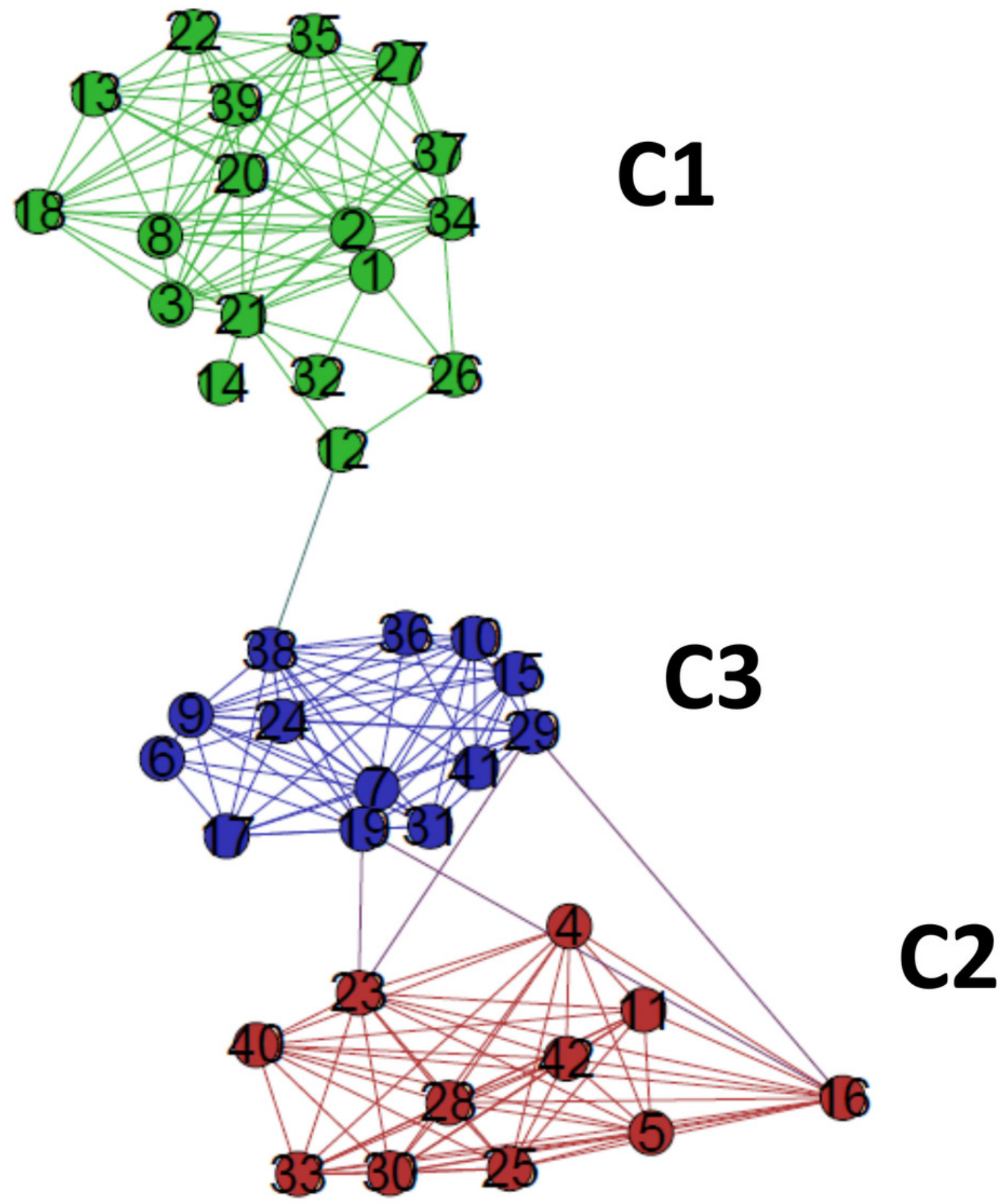


Figure 8 (on next page)

Dendrogram produced by the successive elimination of edges with the largest value of betweenness. Bootstrap values for all branches were produced according to the method described in the text.

Bootstrap support for communities AB (C3 + C2), A (C3), B (C2), and C (C1) in complex network method. (Note: The terminology $A, B$ and $C$ is those used in tree-based methods). 


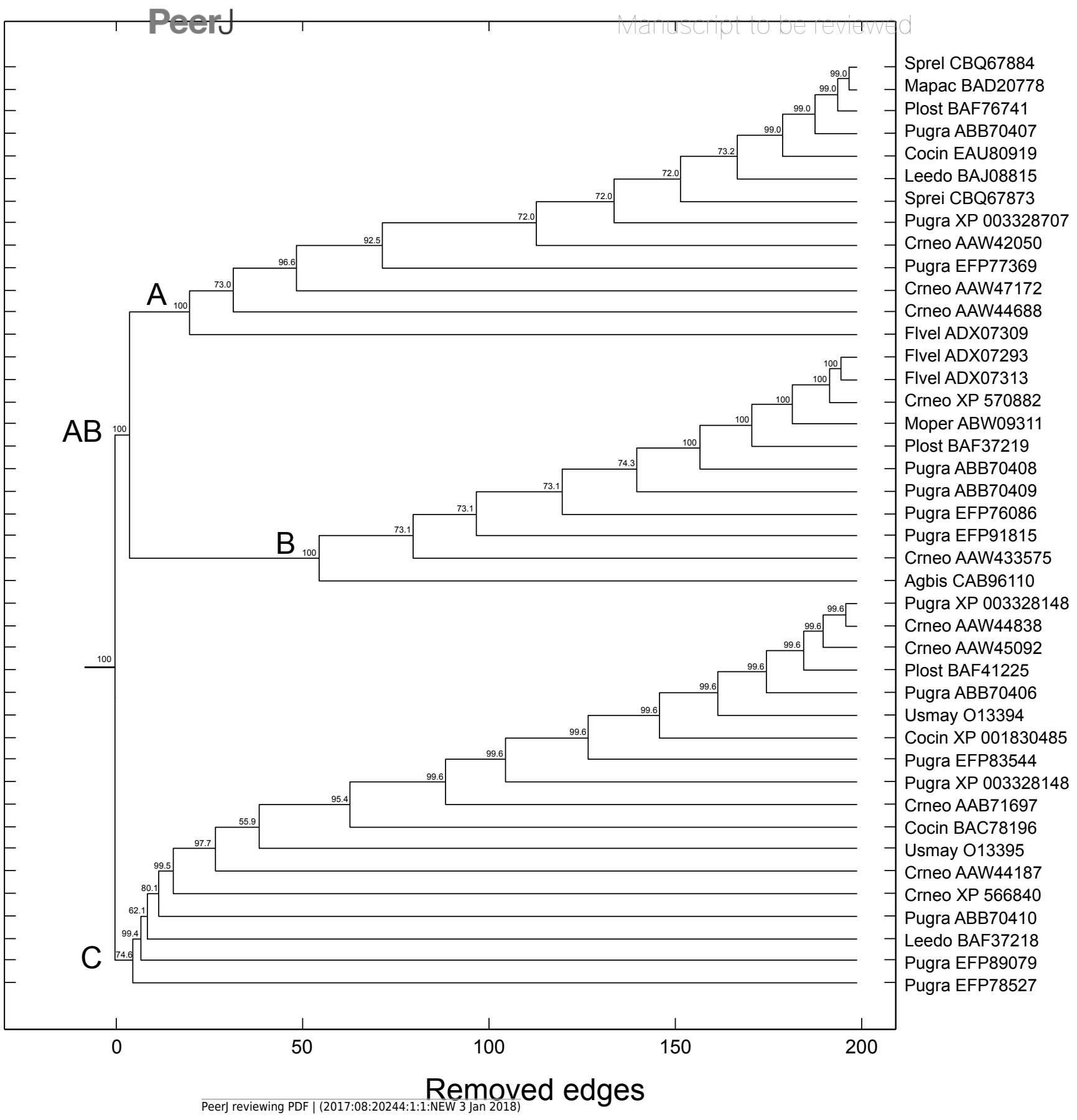




\section{Table $\mathbf{1}$ (on next page)}

Description of the most usual indices and functions used to characterize a network.

A brief description of the meaning of indices and functions used to characterize the structure of complex networks. 
1 Table 1: Description of the most usual indices and functions used to characterize a network.

\begin{tabular}{|c|c|c|}
\hline Symbol & Denomination & Description \\
\hline$N$ & number of nodes & $\begin{array}{l}\text { Number of nodes in the network. } N(N-1) / 2 \text { is the } \\
\text { maximal number of edges, or connections, the network } \\
\text { may contain. }\end{array}$ \\
\hline$k_{i}$ & node degree & $\begin{array}{l}\text { Number of neighbors of node } i \text {, i.e., nodes connected to } \\
\text { node } i \text { by an edge. }\end{array}$ \\
\hline$c_{i}$ & $\begin{array}{l}\text { node clustering } \\
\text { coefficient }\end{array}$ & $\begin{array}{l}\text { Number of edges between the neighbors of node } i \\
\text { divided by } k_{i}\left(k_{i}-1\right) / 2 \text {. }\end{array}$ \\
\hline$d_{i j}$ & $\begin{array}{l}\text { shortest path between } \\
\text { two nodes }\end{array}$ & $\begin{array}{l}\text { Smallest number of edges necessary to connect two } \\
\text { nodes } i \text { and } j \text {. }\end{array}$ \\
\hline$b^{n}{ }_{i}$ & node betweenness & $\begin{array}{l}\text { Number of shortest paths between all pairs of nodes that } \\
\text { go through node } i \text { divided by } N(N-1) / 2 \text {. }\end{array}$ \\
\hline$b_{i j}^{e}$ & edge betweenness & $\begin{array}{l}\text { Number of shortest paths between all pairs of nodes that } \\
\text { go through node } i \text { divided by } N(N-1) / 2 \text {. }\end{array}$ \\
\hline$q_{i}$ & degree assortativity & $\begin{array}{l}\text { Measure of the average degree of the neighbors of node } i \\
\text { as compared to the degree } k_{i} \text {. It assumes values between } \\
+1 \text { and }-1 \text {. The extreme values indicate whether the } \\
\text { neighbors of } i \text { have degree close or distant from } k_{i} \text {. }\end{array}$ \\
\hline$<k>$ & average degree & Average value of $k_{i}$ taken over all $N$ nodes. \\
\hline$C$ & $\begin{array}{l}\text { network clustering } \\
\text { coefficient }\end{array}$ & Average value of $c_{i}$ taken over all $N$ nodes. \\
\hline$<d>$ & average shortest path & Average value of $d_{i j}$ taken over all $N$ nodes. \\
\hline$B_{n}$ & $\begin{array}{l}\text { average node } \\
\text { betweenness }\end{array}$ & Average value of $b^{n}{ }_{i}$ taken over all $N$ nodes. \\
\hline$B_{e}$ & $\begin{array}{l}\text { average edge } \\
\text { betweenness }\end{array}$ & Average value of $b_{i j}^{e}$ taken over all $N$ nodes. \\
\hline$Q$ & $\begin{array}{l}\text { average degree } \\
\text { assortativity }\end{array}$ & Average value of $q_{i}$ taken over all $N$ nodes. \\
\hline$D$ & network diameter & Largest value of $d_{i j}$ \\
\hline$p(k)$ & $\begin{array}{l}\text { probability distribution } \\
\text { of nodes with } k \text { links }\end{array}$ & $\begin{array}{l}\text { Characterizes one important aspect of network related to } \\
\text { the presence or not of hubs (nodes with very large degree } \\
\text { in comparison to }\langle k>\text {. }\end{array}$ \\
\hline$C(k)$ & $\begin{array}{l}\text { probability distribution } \\
\text { of } c_{i} \text { with respect to } k_{i} \text {. }\end{array}$ & $\begin{array}{l}\text { Characterizes the correlation between the values of } k_{i} \\
\text { and } c_{i} \text {. }\end{array}$ \\
\hline$d_{b}$ & fractal dimension & $\begin{array}{l}\text { Measure of invariance of the distribution of nodes. } \\
\text { Relevant when the network admits a cascade of } \\
\text { substructures inside similar substructures. }\end{array}$ \\
\hline$m_{d}$ & modularity & $\begin{array}{l}\text { Measure of the presence of modules or communities. } \\
\text { These are subsets of nodes such that the number of edges } \\
\text { between them is much larger than the number of edges } \\
\text { linking this subset to other nodes outside it. }\end{array}$ \\
\hline
\end{tabular}




\section{Table 2 (on next page)}

List of the basidiomycotan chitin synthase sequences used in this work.

List of the basidiomycotan chitin synthase sequences and their corresponding qualiquantitative features and assignment to the groups retrieved in phylogenetic tree-based and network-based analyses. 


\begin{tabular}{|c|c|c|c|c|c|c|c|c|c|c|c|}
\hline $\begin{array}{l}\text { Id. } \\
\text { No. }\end{array}$ & $\begin{array}{l}\text { Id. Protein } \\
\text { (NCBI) }\end{array}$ & $\begin{array}{l}\text { Species } \\
\end{array}$ & $\begin{array}{c}\text { No. amino } \\
\text { acid } \\
\text { residues }\end{array}$ & $\begin{array}{c}\mathrm{MW} \\
(\mathrm{kDa})\end{array}$ & $\mathrm{pl}$ & $\begin{array}{c}\text { Transmembrane } \\
\text { regions profile }\end{array}$ & $\begin{array}{l}\mathrm{CDD} \\
\text { profile }\end{array}$ & Conserved domains & $\begin{array}{c}\text { CHS class } \\
\text { (Goncalves et al., } \\
\text { 2016) } \\
\end{array}$ & $\begin{array}{l}\text { Group in } \\
\text { Phylogenetic } \\
\text { Analyses } \\
\end{array}$ & $\begin{array}{c}\text { Community in } \\
\text { Complex network } \\
\text { analysis }\end{array}$ \\
\hline 6 & AAW44688 & $\begin{array}{l}\text { Cryptococcus neoformans var. neoformans } \\
\text { JEC22 } \\
\text { Cryptococcus neoformans var. neoformans }\end{array}$ & 947 & 106.385 & 8.86 & 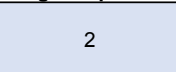 & 2 & PF01644 PF08407 & 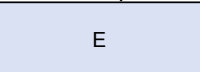 & A & C3 \\
\hline 7 & AAW42050 & $J E C 21$ & 1024 & 112.904 & 8.20 & 2 & 2 & PF01644 PF08407 & B & A & C3 \\
\hline 9 & XP 003328707 & Puccinia graminis f. sp. tritici CRL 75-36-700-3 & 910 & 102.931 & 6.96 & 2 & 2 & PF01644 PF08407 & B & A & C3 \\
\hline 10 & $\underline{\mathrm{CBQ} 67873}$ & Sporisorium reilianum & 940 & 104.729 & 7.07 & 2 & 2 & PF01644 PF08407 & B & A & C3 \\
\hline 15 & EFP77369 & Puccinia graminis f. sp. tritici CRL 75-36-700-3 & 891 & 99.563 & 8.27 & 2 & 2 & PF01644 PF08407 & c & A & C3 \\
\hline 17 & BAJ08815 & Lentinula edodes & 866 & 97.134 & 8.37 & 2 & 2 & PF01644 PF08407 & $\mathrm{E}$ & A & C3 \\
\hline 19 & EAU80919 & Coprinopsis cinerea okayama7\#130 & 941 & 105.442 & 7.61 & 2 & 2 & PF01644 PF08407 & B & A & C3 \\
\hline 24 & $\underline{\mathrm{ABB} 70407}$ & Puccinia graminis f. sp. tritici & 919 & 104.076 & 7.84 & 2 & 2 & $\begin{array}{l}\text { PF01644 PF08407 } \\
\text { PF01644 PF08407 }\end{array}$ & B & A & $\mathrm{C} 3$ \\
\hline 29 & BAF76741 & Pleurotus ostreatus & 927 & 103.784 & 6.92 & 2 & 2 & PF03142 & B & A & C3 \\
\hline 31 & BAD20778 & $\begin{array}{l}\text { Malassezia pachydermatis } \\
\text { Cryptococcus neoformans var. neoformans }\end{array}$ & 805 & 91.459 & 8.64 & 2 & 2 & PF01644 PF08407 & B & A & C3 \\
\hline 36 & AAW47172 & JEC21 & 996 & 110.026 & 7.26 & 2 & 2 & PF01644 PF08407 & C & A & C3 \\
\hline 38 & CBQ67884 & Sporisorium reilianum & 977 & 108.015 & 6.53 & 2 & 2 & $\begin{array}{l}\text { PF01644 PF08407 } \\
\text { PF01644 PF08407 }\end{array}$ & c & A & C3 \\
\hline 41 & ADX07309 & Flammulina velutipes & 1676 & 185.073 & 6.00 & 7 & 2 & PF03142 & C & A & $\mathrm{C} 3$ \\
\hline 4 & CAB96110 & $\begin{array}{l}\quad \text { Agaricus bisporus } \\
\text { Cryptococcus neoformans var. neoformans }\end{array}$ & 909 & 102.259 & 8.45 & 2 & 2 & PF01644 PF08407 & III & B & $\mathrm{C} 2$ \\
\hline 5 & AAW43575 & JEC21 & 931 & 104.441 & 8.38 & 2 & 2 & PF01644 PF08407 & III & B & $\mathrm{C} 2$ \\
\hline 11 & EFP91815 & Puccinia graminis f. sp. tritici CRL 75-36-700-3 & 870 & 97.036 & 8.97 & 2 & 2 & PF01644 PF08407 & III & B & C2 \\
\hline 16 & EFP76086 & Puccinia graminis f. sp. tritici CRL 75-36-700-3 & 969 & 108.438 & 7.03 & 2 & 2 & PF01644 PF08407 & III & B & $\mathrm{C} 2$ \\
\hline 23 & ABB70409 & Puccinia graminis f. sp. tritici & 977 & 109.334 & 6.90 & 2 & 2 & PF01644 PF08407 & III & B & $\mathrm{C} 2$ \\
\hline 25 & ABB70408 & Puccinia graminis $f$. sp. tritici & 868 & 96.632 & 8.85 & 2 & 2 & PF01644 PF08407 & III & B & $\mathrm{C} 2$ \\
\hline 28 & BAF37219 & Pleurotus ostreatus & 938 & 105.202 & 8.62 & 2 & 2 & PF01644 PF08407 & III & B & $\mathrm{C} 2$ \\
\hline 30 & ABW09311 & $\begin{array}{l}\text { Moniliophthora perniciosa } \\
\text { Cryptococcus neoformans var. neoformans }\end{array}$ & 913 & 102.762 & 8.68 & 2 & 2 & PF01644 PF08407 & III & B & C2 \\
\hline 33 & XP 570882 & JEC21 & 755 & 85.383 & 8.74 & 2 & 2 & $\begin{array}{l}\text { PF01644 PF08407 } \\
\text { PF01644 PF08407 }\end{array}$ & III & B & $\mathrm{C} 2$ \\
\hline 40 & ADX07313 & Flammulina velutipes & 620 & 70.162 & 9.30 & 6 & 2 & $\begin{array}{c}\text { PF03142 } \\
\text { PF01644 PF08407 }\end{array}$ & III & B & $\mathrm{C} 2$ \\
\hline 42 & ADX07293 & $\begin{array}{l}\text { Flammulina velutipes } \\
\text { Cryptococcus neoformans var. neoformans }\end{array}$ & 864 & 97.184 & 7.57 & 2 & 2 & PF03142 & III & B & $\mathrm{C} 2$ \\
\hline 1 & $\underline{X P 566840}$ & $J E C 21$ & 1271 & 143.041 & 5.46 & 1 & 1 & PF03142 & $\mathrm{Vb}$ & c & C1 \\
\hline 2 & $\underline{\mathrm{AAB} 71697}$ & Cryptococcus neoformans var. grubii & 1041 & 116.201 & 8.84 & 1 & 1 & PF03142 & $\mathrm{IVb}$ & c & C1 \\
\hline 3 & BAC78196 & Coprinopsis cinerea & 1409 & 157.676 & 8.92 & 1 & 1 & PF03142 & IVa & c & C1 \\
\hline 8 & $\underline{X P 003328148}$ & Puccinia graminis f. sp. tritici CRL 75-36-700-3 & 1417 & 155.072 & 7.41 & 4 & 1 & PF03142 & IVa & c & C1 \\
\hline 12 & EFP89079 & Puccinia graminis f. sp. tritici CRL 75-36-700-3 & 1729 & 192.445 & 6.28 & 3 & 3 & PF03142 PF00063 & $\mathrm{Va}$ & c & C1 \\
\hline 13 & EFP83544 & Puccinia graminis f. sp. tritici CRL 75-36-700-3 & 1212 & 135.277 & 8.28 & 1 & 1 & PF03142 & $\mathrm{IVb}$ & c & C1 \\
\hline 14 & EFP78527 & Puccinia graminis f. sp. tritici CRL 75-36-700-3 & 2066 & 227.286 & 5.63 & 3 & 3 & PF03142 PF00063 & $\mathrm{Vb}$ & c & C1 \\
\hline 18 & $\underline{X P 001830485}$ & Coprinopsis cinerea okayama7\#130 & 1147 & 125.865 & 8.77 & 1 & 1 & PF03142 & $\mathrm{IVb}$ & c & C1 \\
\hline 20 & AAB84284 & Ustilago maydis & 1486 & 162.724 & 9.00 & 1 & 1 & PF03142 & IVa & c & C1 \\
\hline 21 & AAB84285 & Ustilago maydis & 1180 & 130.623 & 6.07 & 1 & 1 & PF03142 & $\mathrm{Vb}$ & c & C1 \\
\hline 22 & ABB70406 & Puccinia graminis f. sp. tritici & 1019 & 113.685 & 9.02 & 1 & 1 & PF03142 & $\mathrm{IVb}$ & c & C1 \\
\hline 26 & ABB70410 & Puccinia graminis & 1997 & 222.514 & 6.44 & 3 & 3 & PF03142 PF00063 & $\mathrm{Va}$ & c & C1 \\
\hline 27 & BAF41225 & Pleurotus ostreatus & 1436 & 159.927 & 8.44 & 1 & 1 & PF03142 & IVa & $\mathrm{c}$ & $\mathrm{C} 1$ \\
\hline 32 & BAF37218 & Lentinula edodes & 1937 & 215.318 & 5.42 & 3 & 3 & PF03142 PF00063 & $\mathrm{Vb}$ & C & C1 \\
\hline 34 & AAW45092 & Cryptococcus neoformans var. neoformans & 1423 & 158.113 & 7.85 & 1 & 1 & PF03142 & IVa & C & C1 \\
\hline
\end{tabular}




\begin{tabular}{|c|c|c|c|c|c|c|c|c|c|c|c|}
\hline 35 & AAW44838 & $\begin{array}{c}\text { Cryptococcus neoformans var. neoformans } \\
\text { JEC } 21 \\
\text { Cryptococcus neoformans var. neoformans }\end{array}$ & 1236 & 136.325 & 8.77 & 1 & 1 & PF03142 & $\mathrm{IVb}$ & c & C1 \\
\hline 37 & AAW44187 & JEC21 & 1895 & 214.398 & 6.41 & 3 & 3 & PF03142 PF00063 & $\mathrm{Va}$ & C & C1 \\
\hline 39 & XP 003327390 & Puccinia graminis f. sp. tritici CRL $75-36-700-3$ & 519 & 58.518 & 7.16 & 5 & 1 & PF03142 & $\mathrm{IVb}$ & c & C1 \\
\hline
\end{tabular}




\section{Table 3 (on next page)}

Comparison of the support indexes of the tree-based methods.

Comparison of the support indexes (bootstrap and posterior probabilities) of the tree-based methods. 
1 Table 3. Comparison of the support indexes of the tree-based methods.

2

\begin{tabular}{ccccc}
\hline Groups & $\begin{array}{c}\text { Maximum } \\
\text { Parsimony } \\
(\mathbf{\%} \text { BP) }\end{array}$ & $\begin{array}{c}\text { Distance } \\
(\mathbf{\%} \text { BP) }\end{array}$ & $\begin{array}{c}\text { Maximum Likelihood } \\
(\%) ~ B P)\end{array}$ & $\begin{array}{c}\text { Bayesian } \\
(\% \mathbf{P P})\end{array}$ \\
\hline $\mathrm{AB}$ & 100 & 100 & 100 & 100 \\
$\mathrm{C}$ & $<50$ & $<50$ & 100 & $<50$ \\
$\mathrm{~A}$ & 54 & 100 & $<50$ & 93 \\
$\mathrm{~B}$ & 100 & 100 & 97 & 100 \\
\hline
\end{tabular}




\section{Table 4 (on next page)}

Comparison of the number of removed edges and support indexes of tree-based and complex networks methods.

Comparison of the number of removed edges and support indexes (bootstrap and posterior probabilities) of tree-based and complex networks methods. 
1 Table 4. Comparison of the number of removed edges and support indexes of tree-based and 2 complex networks methods.

3

\begin{tabular}{lccccc}
\hline Groups & Complex Networks & Maximum Parsimony & Distance & $\begin{array}{c}\text { Maximum } \\
\text { Likelihood }\end{array}$ & Bayesian \\
\hline $\mathrm{A}+\mathrm{B}=$ & 5 & 100 & 100 & 100 & 100 \\
$\mathrm{C} 3+\mathrm{C} 2$ & 6 & $<50$ & $<50$ & 100 & $<50$ \\
$\mathrm{C}=\mathrm{C} 1$ & 54 & 100 & $<50$ & 93 \\
$\mathrm{~A}=\mathrm{C} 3$ & 21 & 100 & 100 & 100 & 100 \\
$\mathrm{~B}=\mathrm{C} 2$ & 75 & & & \\
\hline
\end{tabular}

